\title{
Static Recipient Cells as Reservoirs of Antibiotic Resistance During Antibiotic Therapy
}

\author{
Allan R. Willms* \\ Dept. of Mathematics and Statistics, University of Guelph, Guelph, ON, N1G 2W1, Canada \\ Paul D. Roughan \\ School of Biological Sciences and New Zealand Institute of Gene Ecology, University of \\ Canterbury, Christchurch, New Zealand \\ Jack A. Heinemann \\ School of Biological Sciences and New Zealand Institute of Gene Ecology, University of \\ Canterbury, Christchurch, New Zealand \\ Postprint of article published in: Theor. Pop. Biol. 70 (4) (2006) 436-451.
}

March 28, 2006

\begin{abstract}
How does taking the full course of antibiotics prevent antibiotic resistant bacteria establishing in patients? We address this question by testing the possibility that horizontal/lateral gene transfer (HGT) is critical for the accumulation of the antibioticresistance phenotype while bacteria are under antibiotic stress. Most antibiotics prevent bacterial reproduction, some by preventing de novo gene expression. Nevertheless, in some cases and at some concentrations, the effects of most antibiotics on gene expression may not be irreversible. If the stress is removed before the bacteria are cleared from the patients by normal turnover, gene expression restarts, converting the residual population to phenotypic resistance. Using mathematical models we investigate how static recipients of resistance genes carried by plasmids accumulate resistance genes, and how specifically an environment cycling between presence and absence of the antibiotic uniquely favors the evolution of horizontally mobile resistance genes. We found that the presence of static recipients can substantially increase the persistence of the plasmid and that this effect is most pronounced when the cost of carriage of the plasmid decreases the cell's growth rate by as much as a half or more. In addition, plasmid
\end{abstract}

${ }^{*}$ Corresponding author. 
persistence can be enhanced even when conjugation rates are as low as half the rate required for the plasmid to persist as a parasite on its own.

Keywords: Antibiotic resistance, Plasmid persistence, Horizontal gene transfer, Differential equations

\section{Introduction}

Doctors often tell you to complete your full course of prescribed antibiotics, otherwise, they warn, you increase the chances of another infection that is also more likely to be resistant to the antibiotic. But how is this so? How does the premature cessation of antibiotic use cause populations of bacteria to become resistant to the antibiotic at the scale of individual patients?

Bacteria with pre-existing resistance phenotypes would probably have survived the antibiotic anyway, so the implication is that by taking the antibiotic for a short time, the patient has been made vulnerable to colonization by resistant strains, or the susceptible bacteria acquired resistance during therapy. It remains a mystery, however, why in these cases more of the same antibiotic is protective.

We developed mathematical models based on our previous experimental demonstrations of the effects of antibiotics on horizontal gene transfer by bacterial conjugation, to determine whether conditions specific to abortive antibiotic therapy favored the short-term evolution of resistant strains in patients. Certain infectious agents, called conjugative plasmids, continue to reproduce infectiously, that is, by horizontal gene transfer, despite application of many types of antibiotics to bacteria (Cooper and Heinemann, 2000b; Heinemann, 2000; Heinemann and Ankenbauer, 1993a,b; Heinemann et al., 1996). Based on these empirical results, we hypothesized that antibiotics were particularly effective at biasing the evolution of resistance genes to accumulate on plasmids (Heinemann, 1999; Heinemann and Silby, 2003), at least in the early stages of antibiotic selection, because the reproduction of the bacterial genome (i.e., the chromosome) would be slower than that of the plasmid. Differential reproductive rates between chromosomes and plasmids have long been used to explain why some genes accumulate on different types of replicons (Eberhard, 1989; Heinemann, 1998; Souza and Eguiarte, 1997).

Balancing the effectiveness of plasmid transfer is the cost of plasmid carriage or the resistance phenotype itself, especially in the absence of the drug (Gillespie and McHugh, 1997). While these costs may be substantial, there is doubt as to their long-term relevance in nature because compensatory adaptations emerge rapidly (Heinemann et al., 2000; Lenski et al., 1994; Modi et al., 1992; Schrag and Perrot, 1996; Schrag et al., 1997). Still, these costs may be important early in the relationship between resistance gene and host.

Our model relies on the establishment of a unique cell physiology during antibiotic therapy. That physiology is characterized by the inability to reproduce but the maintenance of 
most biochemical pathways in the cell. We call this type of a cell "dead" with reference to its potential to reproduce (in the microbiological sense) but it remains alive as a recipient and perhaps donor of genes (Heinemann, 1998). This reservoir of gene vectors has never to our knowledge been incorporated into models of resistance evolution before.

Most antibiotics prevent bacterial reproduction, some by preventing de novo gene expression. Nevertheless, in some cases and at some concentrations, the effects of most antibiotics on gene expression may not be irreversible (Wiuff et al., 2005). If the stress is removed before the bacteria are cleared from the patients by normal turnover, gene expression restarts, converting the residual population to phenotypic resistance.

Plasmids are ubiquitous horizontally mobile elements (HMEs) in natural bacterial populations. They can both transfer vertically, at cell division, and they can transfer horizontally, most commonly by conjugation (Frost, 2000; Heinemann, 1991). Plasmids that establish within populations often carry genes that adapt the host cell to its current environment, such as genes coding for antibiotic resistance. Even plasmids that confer no detectable adaptive benefit upon the host may persist, possibly due to so-called "addiction" or post-segregational killing systems (Cooper and Heinemann, 2000a, 2005; Gerdes et al., 1986).

Post-segregational killing systems (PSK) are ubiquitous on natural plasmids. The system effects a high efficiency of cell death under conditions where continued gene expression is reduced or prevented, such as when a plasmid mis-segregates to only one daughter during cell division. PSK systems are biochemically diverse and range from DNA restriction-modification systems to RNA molecules that are antisense to the mRNA of a toxin (Kobayashi, 1998). In all cases, the common attribute of PSK systems is that they coordinately produce a stable toxin and less stable antitoxin; when the stoichiometry between these molecules changes for whatever reason, the more stable toxin can achieve toxic concentrations and kill the cell (Cooper and Heinemann, 2000a, 2005).

The effect of PSK on stable monocultures of bacteria is to stabilize the plasmid in the population despite the cost of plasmid carriage (Cooper and Heinemann, 2000a). Bacteria under competition, or plasmid under competition from other infectious elements such as other plasmids and bacteriophage, however, are at a disadvantage if the competitors have no PSK systems (Cooper and Heinemann, 2000a). A competitive advantage is fully restored so long as the PSK system is horizontally mobile, such as by residing on a conjugative plasmid (Cooper and Heinemann, 2000a). Under these circumstances, PSK systems eliminate other infectious elements by preventing their establishment in the population. PSK therefore contributes to plasmid persistence both by driving dispersal of the plasmid and the stability of the plasmid during vertical growth of a population.

Bergstrom et al. (2000) addressed the question of persistence under the assumption that conjugation rates are too low to allow the plasmid to persist simply as a parasite. That is, they limited themselves to the case where the cost of plasmid carriage and the loss due to missegregation during cell division always outweigh the gains from conjugation. Under this 
situation, the plasmid can clearly only persist if it carries genes that are of benefit to the host.

The assumption that conjugation rates are never high enough to compensate for carriage and missegregation losses is of course questionable. If conjugation were modeled as proportional to the product of donor and recipient cells, as is usual (Levin et al., 1979; Simonsen, 1991; Smith, 2001; Dionisio et al., 2002; Imran et al., 2005), then such an assumption necessarily implies an environmentally imposed limit on the size of the bacterial populations. If unlimited growth were allowed, conjugation rates would eventually outstrip carriage and missegregation losses and the plasmid could persist. Models such as that by Smith (2001) explain plasmid persistence in this manner. Most conjugation rates have been measured for cells in liquid culture rather than biofilms or microcolonies where natural bacterial populations are regularly found and in which conjugation rates could be higher. Even so, reported conjugation rates vary widely (Gordon, 1992; Dionisio et al., 2002). Dionisio et al. (2002) report a very large variation in "donor-ability" of bacteria and suggest that plasmids could spread quickly through heterogeneous populations once they transfer to a strain that has a high donor capacity. In addition, carriage costs and missegregation losses are clearly tied to cell growth whereas conjugation frequency is not necessarily proportional to cell growth. In situations of severely restricted resource, it is not difficult to imagine carriage costs and missegregation losses dropping to a level below the gains from conjugation. Indeed conjugation can continue to occur even when growth is stopped (Heinemann, 1999; Peters and Benson, 1995).

Even without possibly enhanced conjugation rates within biofilms, the physical properties of the biofilm itself may enhance plasmid persistence within such structures (Imran et al., 2005). Interestingly, Ghigo (2001) has shown that conjugative plasmids actually encourage biofilm development. S. Turner et al. (2002) argue that environment is extremely important for the maintenance of HMEs and suggest that simple mass-action models of conjugation will not likely capture the full range of phenomena in diverse structured communities. In particular, P. Turner (2004) provides evidence that conjugation rates for plasmid pB15 in $E$. coli in static (not well-mixed) environments do not increase in proportion to cell density. Nonetheless, models of this sort are beneficial in aiding our understanding of HME maintenance and can go a long way to explaining some of the features of HME dynamics (Levin et al., 1979; Simonsen, 1991).

In this work, we focus on modeling the dynamics associated with plasmids carrying resistance genes for chloramphenicol (CM). Chloramphenicol is a bacteriostatic agent which prevents cell division through the inhibition of protein synthesis. The chloramphenicol molecule binds reversibly to the peptidyl-amyltransferase enzyme and prevents peptide chain elongation (Amábile-Cuevas, 1996). On removal of the antibiotic, static cells are able to resume growth. If some bacteria in a population carry a plasmid encoding a gene or genes which confer resistance to $\mathrm{CM}$, then the question arises as to whether conjugation events can occur between plasmid-bearing CM-resistant cells and plasmid-free cells that have been arrested 
in their growth cycle by the presence of CM. If conjugation does occur while CM is present, then it is not unreasonable to assume that although the recipient cell now harbours the resistance-bearing plasmid, it is still unable to synthesize proteins and therefore cannot express the resistance genes. The recipient cell would in this case be genotypically resistant but phenotypically equivalent to static cells harbouring no plasmid at all. Here, using mathematical models, we study how the inclusion of this mechanism can enhance the persistence and relative frequency of CM resistance-bearing plasmids in environments where CM is periodically applied and removed even if conjugation rates are too low to allow the plasmid to persist in the continued absence of selection.

\section{Model}

Our model of plasmid population dynamics is based on one by Stewart and Levin (1977) for a single clonal bacterial cell population with one plasmid type and a single limiting resource $(R)$, and is informed by empirical measures published by this group over the last ten years (Cooper and Heinemann, 2000b, 2005; Heinemann, 2000; Heinemann and Ankenbauer, 1993a,b; Heinemann et al., 1996). Plasmid-free cells $(F)$ carry no copy of the CM resistance gene and are sensitive to $\mathrm{CM}$ action. Plasmid-bearing cells $(P)$ carry a copy of the plasmid and exhibit resistance to $\mathrm{CM}$. We do not model plasmid copy number and assume that all plasmid-bearing cells are equally effective at resisting CM. We assume the introduction and removal of chloramphenicol occurs instantaneously and that plasmid-bearing cells are completely unaffected by CM presence while plasmid-free cells cease all growth in the presence of CM. These last assumptions may be relaxed without qualitative changes in our results. When CM is present, conjugation of plasmid-bearing cells with plasmid-free cells results in the formation of a new class of cells $\left(F_{+}\right)$which harbour, but do not express the resistance plasmid. Thus these cells, although genotypically identical to $P$ cells, are phenotypically like $F$ cells while CM is present. We assume that $F_{+}$cells are unable to pass on the plasmid via conjugation to other cells. Upon the removal of $\mathrm{CM}, F_{+}$cells immediately become $P$ cells and are capable of being plasmid donors in conjugation events.

The cells inhabit a well-mixed environment through which a constant flow $u>0$ is maintained ( $u$ has units time ${ }^{-1}$ ). Resource at constant concentration $R_{\text {in }}>0$ is present in the input stream. The intrinsic cell growth rate is given by a typical Monod growth function

$$
\psi(R)=g \frac{R}{R_{2}+R},
$$

where $g>0$ is the maximal growth rate and $R_{2}>0$ is the resource level at which the growth rate is half maximal. (We also considered a general Michaelis-Menton growth function of the form

$$
\psi(R)=g \frac{R^{N}}{R_{2}^{N}+R^{N}}
$$


where $N$ is a positive integer but found no significant differences in our results.) Cell death (as opposed to wash out) is not explicitly modeled, but, assuming it is constant, could be absorbed into the flow rate $u$ (with a corresponding decrease in $R_{\text {in }}$ ). Carriage of the plasmid is assumed to reduce the division rate of host cells relative to non-carriers, expressed as a fraction, $\alpha, 0<\alpha<1$, of the normal growth rate. Missegregation events resulting in one plasmid-free daughter cell, will occur at a proportion $\tau, 0 \leq \tau<1$, among plasmid-bearing cell divisions. Our model allows for a PSK mechanism through a parameter $\kappa, 0 \leq \kappa \leq 1$; if $\kappa=1$ PSK is completely effective killing all daughter cells which fail to obtain a copy of the plasmid, while if $\kappa=0$ there is no PSK mechanism and missegregation results in a viable $F$ cell. Plasmid-mediated conjugation is assumed to convert plasmid non-bearers to plasmid bearers at a rate proportional to the product of the donor and recipient cell densities, where the constant of proportionality is $\gamma$.

The system of equations describing these assumptions is

$$
\begin{aligned}
& \text { no CM }\left\{\begin{aligned}
\frac{d P}{d t} & =\underbrace{\psi(R)(1-\alpha)(1-\tau) P}_{\text {growth less carriage \& misseg. }}-\underbrace{u P}_{\text {washout }}+\underbrace{\gamma P F}_{\text {conj. gain }} \\
\frac{d F}{d t} & =\underbrace{\psi(R) F}_{\text {growth }}-\underbrace{u F}_{\text {washout }}-\underbrace{\gamma P F}_{\text {conj. loss }}+\underbrace{\psi(R)(1-\alpha) \tau(1-\kappa) P}_{\text {misseg. gain after PSK }} \\
\frac{d R}{d t} & =\underbrace{R_{\text {in }} u}_{\text {input }}-\underbrace{R u}_{\text {washout }}-\underbrace{\frac{1}{\sigma} \psi(R)(P+F)}_{\text {consumption by } P \text { and } F}
\end{aligned}\right. \\
& \mathrm{CM}\left\{\begin{aligned}
\frac{d P}{d t} & =\psi(R)(1-\alpha)(1-\tau) P-u P+\underbrace{0}_{\text {no conj. }} \\
\frac{d F}{d t} & =\underbrace{0}_{\text {no growth }}-u F-\gamma P F+\psi(R)(1-\alpha) \tau(1-\kappa) P \\
\frac{d F_{+}}{d t} & =-\underbrace{u F_{+}}_{\text {washout }}+\underbrace{\gamma F P}_{\text {conj. gain }} \\
\frac{d R}{d t} & =R_{\text {in } u-R u}-\underbrace{\frac{1}{\sigma} \psi(R) P}_{\text {consumption by } P}
\end{aligned}\right.
\end{aligned}
$$

where $P, F$, and $F_{+}$refer to the biomass of each cell type, $\sigma$ is the yield ratio (biomass of cells per unit resource) and $\psi(R)$ is given by (1). Terms in (2a) and those which differ in $(2 \mathrm{~b})$ are labelled underneath.

If plasmids and chloramphenicol were not present (and provided $u<g$ so that wash out 
does not outstrip growth), $F$ would approach a stable equilibrium value of

$$
K=\sigma\left(R_{\text {in }}-\frac{R_{2}}{\frac{g}{u}-1}\right)
$$

which we define as the carrying capacity of our system. In order to have a positive carrying capacity we further impose

$$
R_{\text {in }}>\frac{R_{2}}{\frac{g}{u}-1}, \quad \text { or, equivalently } \quad \frac{u}{g}<\frac{R_{\text {in }}}{R_{2}+R_{\text {in }}} .
$$

In other words, we do not allow the flow rate to exceed the incoming growth potential which would result in all the cells being washed out of the system. Applying the change of variables

$$
\begin{array}{ll}
\hat{P}=\frac{P}{K}, \quad \hat{F}=\frac{F}{K}, \quad \hat{F}_{+}=\frac{F_{+}}{K}, \quad \hat{R}=\frac{R}{R_{2}}, \hat{t}=g t, \\
\hat{u}=\frac{u}{g}, \quad \hat{R_{\text {in }}}=\frac{R_{\text {in }}}{R_{2}}, \quad \hat{\gamma}=\frac{\gamma K}{g}, \quad \hat{\sigma}=\frac{\sigma R_{2}}{K}=\left(\hat{R_{\text {in }}}-\frac{1}{\frac{1}{\hat{u}}-1}\right)^{-1}
\end{array}
$$

to system (2) gives an equivalent nondimensional system $(\alpha, \tau$, and $\kappa$ are already nondimensional) where the cell populations are measured in fractions of the carrying capacity and the time is measured in units of $e$-fold increase under maximal growth. Effectively, after dropping hats, this new system is identical to (2) with $g$ and $R_{2}$ both replaced by 1 , and $\sigma$ replaced by

$$
\sigma=\left(R_{\mathrm{in}}-\frac{1}{\frac{1}{u}-1}\right)^{-1} .
$$

(Note that (4) implies this nondimensional $\sigma$ is positive.) From now on we analyze system (2) together with (6) and

$$
\psi(R)=\frac{R}{1+R} .
$$

The nondimensional version of (4) is

$$
R_{\text {in }}>\psi^{-1}(u) \quad \text { or, equivalently } u<\psi\left(R_{\text {in }}\right),
$$

with $0<u<1$ where

$$
\psi^{-1}(u)=\frac{1}{\frac{1}{u}-1} .
$$

In order to discern the effect of the $F_{+}$pool of plasmids we compare the model $(2),(6),(7)$ with one where conjugation between plasmid-bearing and plasmid-free cells cannot occur when chloramphenicol is present. This comparison model is identical to the first except that $\gamma$ is set to zero in $(2 \mathrm{~b})$. Thus, $F_{+}$cells are never present. For convenience, we label the model (2),(6),(7) as $M_{+}$and the comparison model as $M_{0}$. 


\section{$2.1 \quad$ Equilibria}

Under no chloramphenicol, the equilibrium point

$$
P=0, \quad F=1, \quad F_{+}=0, \quad R=R_{\text {in }},
$$

is globally (in the nonnegative orthant less the $R$-axis) asymptotically stable provided (8) holds and

$$
\gamma<u[1-(1-\alpha)(1-\tau)]
$$

(See the Appendix.) This last condition states that the plasmid's conjugation advantage does not outweigh its carriage and missegregation costs. In other words, the plasmid cannot exist as a parasite.

With chloramphenicol present, the equilibrium point

$$
\begin{array}{lll}
P=(1-\alpha)(1-\tau) \frac{R_{\text {in }}-\psi^{-1}\left(\frac{u}{(1-\alpha)(1-\tau)}\right)}{R_{\text {in }}-\psi^{-1}(u)}, & F=\frac{(1-\kappa) \tau P}{(1-\tau)\left(1+\frac{\gamma}{u} P\right)}, \\
F_{+}=\frac{\gamma}{u} F P, & R=\psi^{-1}\left(\frac{u}{(1-\alpha)(1-\tau)}\right),
\end{array}
$$

is positive and also appears to be globally asymptotically stable provided

$$
u<(1-\alpha)(1-\tau) \psi\left(R_{\text {in }}\right)
$$

(See the Appendix.) This is a slightly stronger restriction on the washout rate than (8) reflecting the fact that the plasmid-bearing cells do not grow as fast as plasmid-free cells. Note that the equilibrium given by (11) is valid for both models $M_{0}$ and $M_{+}$; for $M_{0}, \gamma$ is zero in the presence of CM.

We impose the restrictions (10) and (12) to ensure that in the absence of selection the plasmid carriers tend to zero while the non-carriers persist, and under selection the plasmid carriers persist.

\subsection{Cycling Environment and Plasmid Success Measure}

We subject our models to a repeated cycle consisting of $T_{n}$ time units during which chloramphenicol is absent (the non-selection regime) followed by $T_{s}$ time units during which chloramphenicol is present (the selection regime). During the non-selection regime of the cycle, the system approaches the equilibrium point (9). At the end of the non-selection regime, the system of governing equations switches from $(2 \mathrm{a})$ to $(2 \mathrm{~b})$ and $F_{+}$is initialized as zero. During the selection regime of the cycle the system approaches the equilibrium point (11), and at the end end of the selection regime, the $F_{+}$cells immediately become $P$ cells yielding a new set of initial conditions for the start of the next cycle governed again by system (2a). 
After a sufficient number of cycles the system approaches a stable oscillation with sharp corners or jumps at the points where chloramphenicol is added or removed. Although conceptually it is possible that the cycling system has more than one attractor, we found no evidence for such; solutions always converged to a unique stable oscillation for any set of positive initial conditions we tried. The long term outcomes of $P$ and $F$ depend on the lengths of the two regimes in the cycle (and on the other parameters in the model). The forced cycling will either drive $P$ to extinction or will result in a situation where the values of $P, F$, and $R$ at the start of each new cycle will approach a set of positive constants. It is impossible for both $P$ and $F$ to be driven to extinction since this would imply that the resource $R$ would approach $R_{\text {in }}$ and at this value $d P / d t>0$ since (12) holds. However, it is possible for both $P$ and $F$ to be driven to very small values. (If the post-segregational killing mechanism is completely effective, $\kappa=1$, then it is possible that $F$ is driven to extinction while $P$ remains positive.) After the stable oscillation had (approximately) been reached, we used the following measure of plasmid success to compare the outcome of models $M_{+}$ and $M_{0}$.

$$
S_{1}=\left.\lim _{k \rightarrow \infty} \frac{P(t)}{P(t)+F(t)}\right|_{t=k\left(T_{n}+T_{s}\right)^{+}}
$$

The statistic $S_{1}$ measures the proportion of plasmid-bearing cells at the start of the cycle. (The superscript plus sign indicates the time of evaluation is after the $F_{+}$cells have been converted to $P$ cells.) We use the notation $S_{1}\left(M_{+}\right)$and $S_{1}\left(M_{0}\right)$ to indicate the success measure evaluated for models $M_{+}$and $M_{0}$ respectively. We also tried a second statistic:

$$
S_{2}=\lim _{k \rightarrow \infty} \frac{\int_{k\left(T_{n}+T_{s}\right)}^{(k+1)} u\left(P+T_{+}\right) d t}{\int_{k\left(T_{n}+T_{s}\right)}^{(k+1)\left(T n+T_{s}\right)} u\left(P+F+F_{+}\right) d t}
$$

which measures the proportion of plasmid-bearing cells exiting from the system over one cycle. However, we found that this second measure gave qualitatively the same, and numerically very similar results to the first, and so only report the first measure herein.

\subsection{Parameter Values and Numerics}

We numerically determined the stable oscillatory solutions of both models over a wide range of parameter values and calculated the plasmid success measure in each case. The parameter values used are given in Table 1. Essentially, each of the model parameters was given a low, medium, and high value within its available range, while the lengths of the non-selection and selection regimes of the cycle were varied from as small as one half to as large as 120 time units. Numerical integration throughout the cycle was done with a local truncation error of $\left(5 \times 10^{-5}\right) / T_{x}$ (relative) and $(5 \times 10-7) / T_{x}$ (absolute) in each component where $x$ is either $n$ or $s$ depending on the regime. Identification of the stable oscillatory solution was achieved 


\begin{tabular}{|c|c|c|}
\hline \multicolumn{2}{|c|}{ parameter and meaning } & values \\
\hline & $\begin{array}{l}\text { length of non-selection regime in } \\
\text { units of } e \text {-fold maximal growth }\end{array}$ & 0.5 or 1 or 2 or 6 or 24 or 120 \\
\hline$T_{s}$ & $\begin{array}{l}\text { length of selection regime in units } \\
\text { of } e \text {-fold maximal growth }\end{array}$ & 0.5 or 1 or 2 or 6 or 24 or 120 \\
\hline$\alpha$ & plasmid carriage cost & 0.1 or 0.5 or 0.95 \\
\hline$\tau$ & missegregation fraction & 0.1 or 0.5 or 0.95 \\
\hline$\kappa$ & PSK efficiency & 0.1 or 0.5 or 0.95 \\
\hline$u$ & $\begin{array}{l}\text { ratio of washout to maximal } \\
\text { growth rates }\end{array}$ & $(0.1$ or 0.5 or 0.95$) \times(1-\alpha)(1-\tau)$ \\
\hline$\gamma$ & $\begin{array}{l}\text { ratio of conjugation rate at carry- } \\
\text { ing capacity to growth rate }\end{array}$ & $(0.1$ or 0.5 or 0.95$) \times u[1-(1-\alpha)(1-\tau)]$ \\
\hline$R_{\text {in }}$ & $\begin{array}{l}\text { input resource concentration in } \\
\text { units of half maximal response }\end{array}$ & $(1.05$ or 2 or 5$) \times \psi^{-1}\left(\frac{u}{(1-\alpha)(1-\tau)}\right)$ \\
\hline
\end{tabular}

Table 1: Nondimensional parameter meanings and values at which success measures were calculated. (The relations between the nondimensional parameters and the dimensional parameters are given by (5).) Every possible arrangement of the values were used, giving a total of $6^{2} \times 3^{6}=26,244$ parameter sets.

with an ad hoc shooting method comparing the state variables at the start of consecutive cycles. Convergence was considered achieved when each component of the difference had a relative error less than $10^{-3}$, or an absolute error less than $10^{-5}$ if the component itself was less than $10^{-2}$. With these tolerances, the error in the success measure, $S_{1}$, is of the order $10^{-3}$ unless $P+F<10^{-2}$, in which case the error could be larger, depending on the relative and absolute errors achieved in the integration of $P$ and $F$. In all cases we estimated these errors and discarded the parameter sets (a total of 473 of the 26,244 sets) for which the estimated error for $S_{1}$ for either model was greater than $10^{-3}$. The maximum and median values for $P$ at the start of the cycle in either model for all these discarded parameter sets were $9.5 \times 10^{-3}$ and $4.3 \times 10^{-4}$ indicating as expected, that large errors in the success measure only occurred when $P$ was very close to extinction and likely zero to within the global numerical integration approximation errors. The total number of parameter sets for which we report results below is $26,244-473=25,771$.

\section{Results}

Some trajectories for models $M_{+}$and $M_{0}$ are shown in Figure 1. The differences between the two models shown in this figure are relatively small, and indeed, over a large part of 

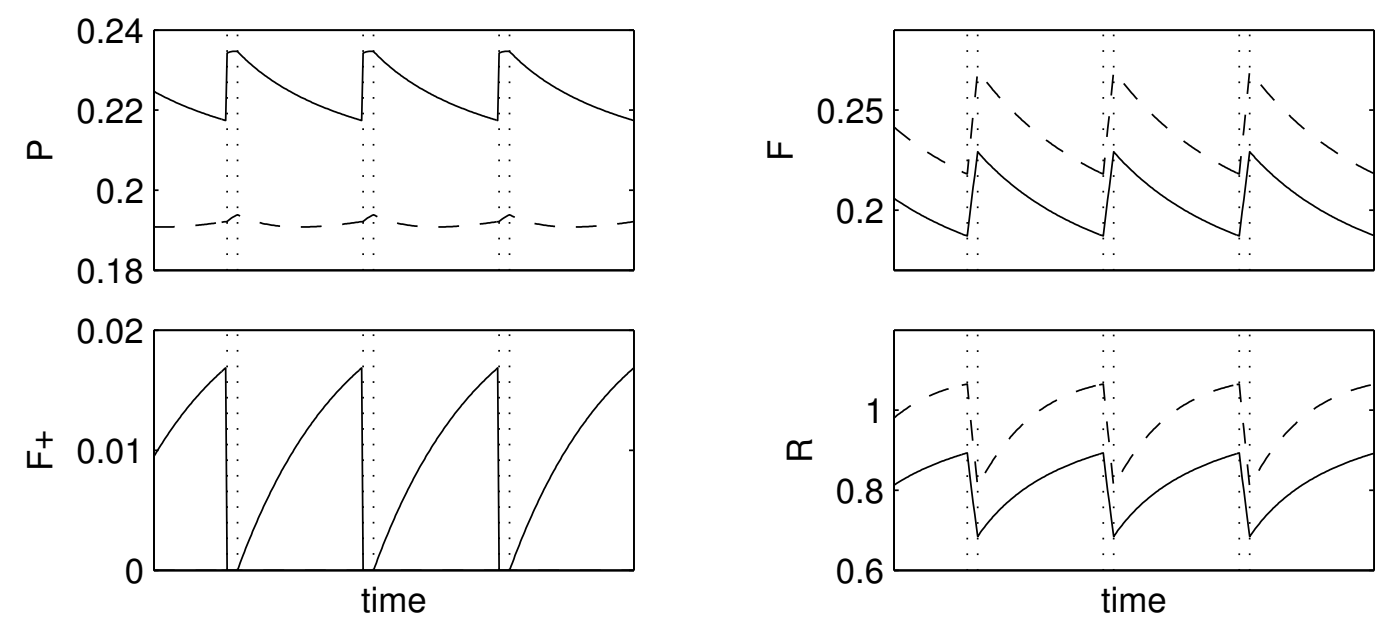

Figure 1: Stable oscillatory solutions of model $M_{+}$(solid lines) and the comparison model $M_{0}$ (dashed lines). The vertical dotted lines are the times at which CM is added or removed; the short time interval is the non-selection regime while the longer is the selection regime. Parameter values are: $T_{n}=0.5, T_{s}=6, \alpha=0.5, \tau=0.5, \kappa=0.1, u=0.5(1-\alpha)(1-\tau)=$ $0.125, \gamma=0.95 u(1-(1-\alpha)(1-\tau))=8.90625 \times 10^{-2}$, and $R_{\text {in }}=5(1 /((1-\alpha)(1-\tau) / u-1))=5$.

the parameter space the differences are zero or very small. The plasmid success measures corresponding to this figure are $S_{1}\left(M_{0}\right)=0.4686$ and $S_{1}\left(M_{+}\right)=0.5559$. However, under certain conditions, the ability for the plasmid to transfer to non-growing cells under $\mathrm{CM}$ selection awards the plasmid a significant advantage.

Figure 2A shows the distribution of plasmid success measures over all of the trial parameter sets for both models. The vast majority of parameter sets gave success measures very near zero, indicating that the plasmid was driven to extinction or to very small values. A sizable portion (about 3,600 of the 25,771 trials) gave success ratios close to one indicating the plasmid was dominant, and intermediate values of the success measure, where plasmidbearing and plasmid-free cells coexist in substantial numbers were rarer. Both models gave very similar distributions of success measure (Figure 2A). We computed the differences in the plasmid success measure for the two models and the distribution of these differences is shown in Figure 2B. In about $13 \%$ of the parameter sets the plasmid success was more that 0.01 higher in model $M_{+}$than in model $M_{0}(3.5 \%$ were more than 0.1 higher $)$. The largest difference we found was 0.507 which occurred when the success measure for $M_{0}$ was about 0.05 . Figure $2 \mathrm{~B}$ also shows that there are situations where the plasmid-bearing cells go extinct (or nearly so) in model $M_{0}$, but including $F_{+}$cells allows plasmid-bearers to comprise up to $50 \%$ of the bacterial population. The number of parameter sets for which the success difference was more than $10^{-2}$ when the success measure for $M_{0}$ was less than $10^{-3}$ 

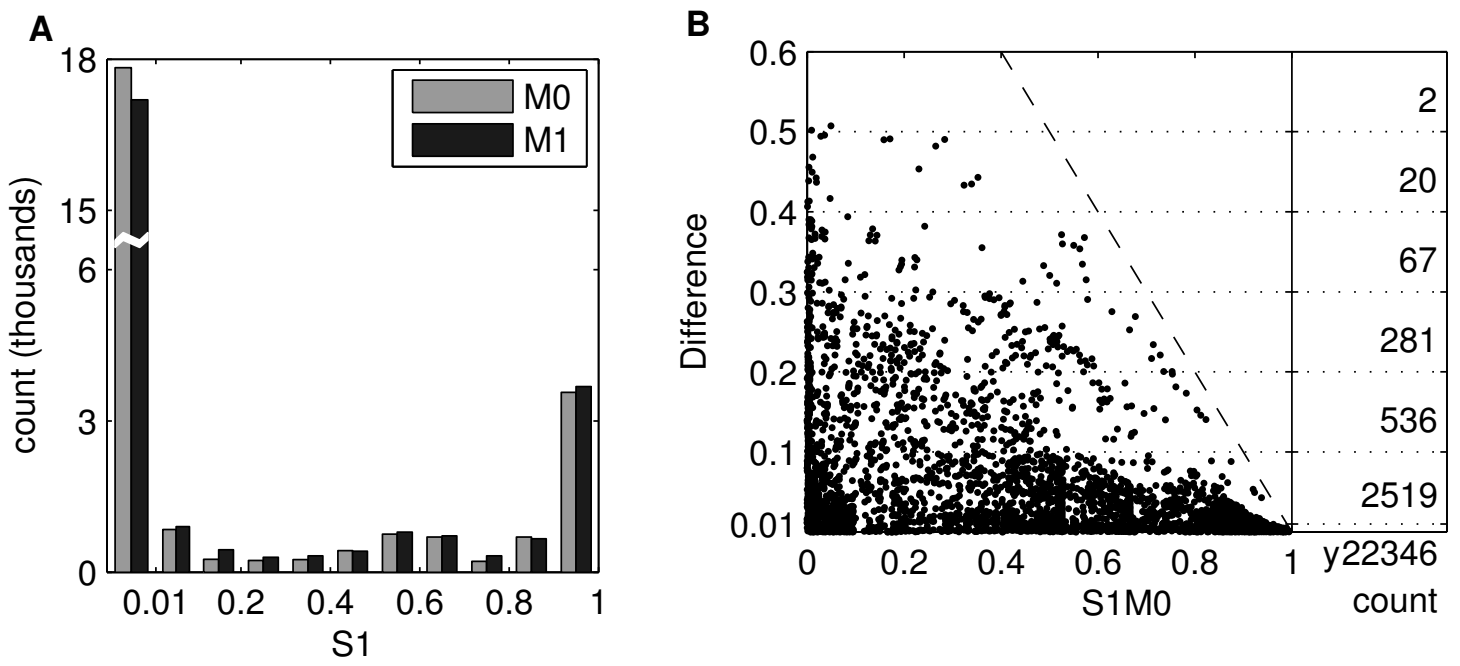

Figure 2: A: Distribution of plasmid success measures, $S_{1}$, for models $M_{0}$ and $M_{+}$. B: The distribution of differences $S_{1}\left(M_{+}\right)-S_{1}\left(M_{0}\right)$ as a function of $S_{1}\left(M_{0}\right)$. The dashed line is the maximum possible difference, since $S_{1}\left(M_{+}\right)$cannot exceed one. The numbers to the right of the graph give the count of events for each range of difference values.

was 360, accounting for about $1.4 \%$ of the total. Since $\tau>0$ and $\kappa<1$, the existence of plasmid-bearing cells implies the existence of some plasmid-free cells also, which is why the data points do not quite reach the dashed line in Figure 2B. Nonetheless, there are clearly some situations where model $M_{0}$ has plasmid frequencies above about $60 \%$ at the start of the cycle while model $M_{+}$has almost exclusively plasmid-bearing cells.

Since $S_{1}$ is a relative measure, it is possible that the largest values of $S_{1}$ correspond to situations where the actual numbers of plasmid-bearing cells is small. Figure 3 shows plots of the success measure as a function of the number of plasmid cells at the beginning of the cycle, for both models. These plots show that over our range of parameter sets, the success measure is well distributed across its possible values. The bottom right triangles of the plots are empty since $P+F \leq 1$ (see the Appendix) which implies $S_{1}>P$.

We analyzed the success measure differences with respect to the relative parameter values. Figures 4-11 show the distribution of success measure differences as a function of $S_{1}\left(M_{0}\right)$ divided into low, medium, and high values for each parameter. In all of these figures, the data from Figure 2B is reproduced but the points corresponding to low, medium, or high values of the named parameter are highlighted. For a given value of $S_{1}\left(M_{0}\right)$, parameter sets which gave the largest values of $S_{1}\left(M_{+}\right)$tended to have high or medium values for $R_{\text {in }}$ (Figure 4) and $\gamma$ (Figure 5), high values for $\kappa$ (Figure 7), and low or medium values for $\tau$ (Figure 8), $\alpha$ (Figure 9), $T_{s}$ (Figure 10), and $T_{n}$ (Figure 11). The conditions on $\kappa$ and $\tau$ 

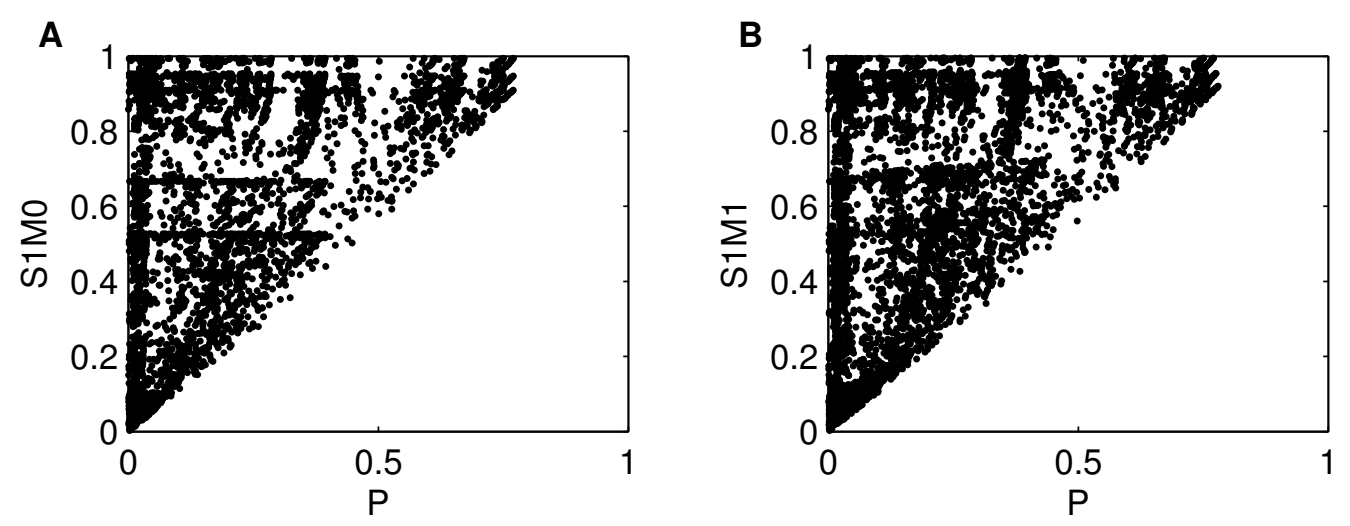

Figure 3: Distribution of success measures as a function of $P$ at the start of the cycle for Model $M_{0}(\mathrm{~A})$ and Model $M_{+}(\mathrm{B})$.
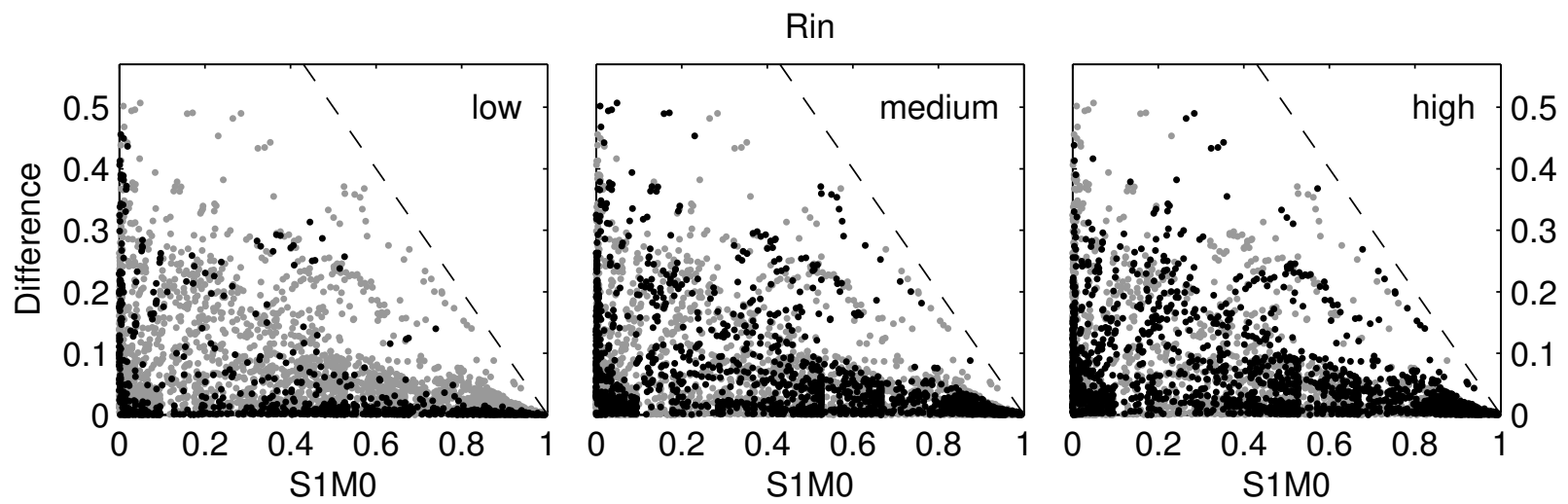

Figure 4: The distribution of differences $S_{1}\left(M_{+}\right)-S_{1}\left(M_{0}\right)$ as a function of $S_{1}\left(M_{0}\right)$ highlighting when $R_{\text {in }}$ has a low (left panel), medium (centre panel), or high (right panel) value. In each plot the black dots are differences where $R_{\text {in }}$ has the indicated value and the lighter dots in the background are the differences for the other values of $R_{\text {in }}$. The dashed line is the maximum possible difference, since $S_{1}\left(M_{+}\right)$cannot exceed one. 


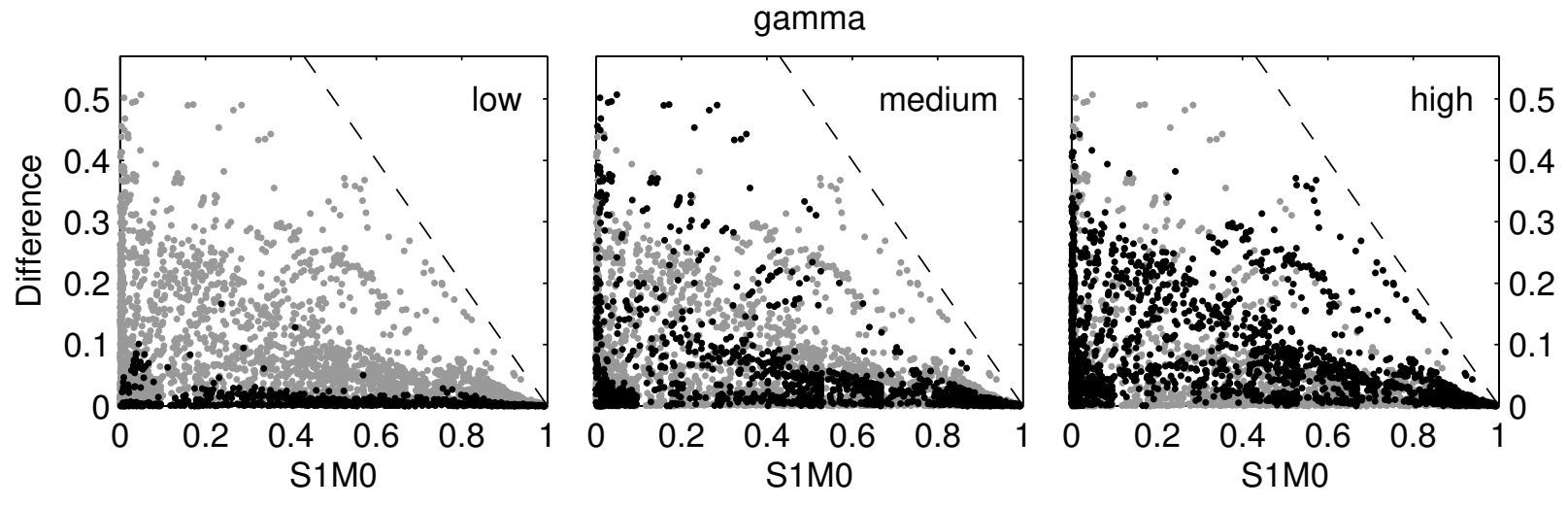

Figure 5: The distribution of differences for parameter $\gamma$. See caption to Figure 4.
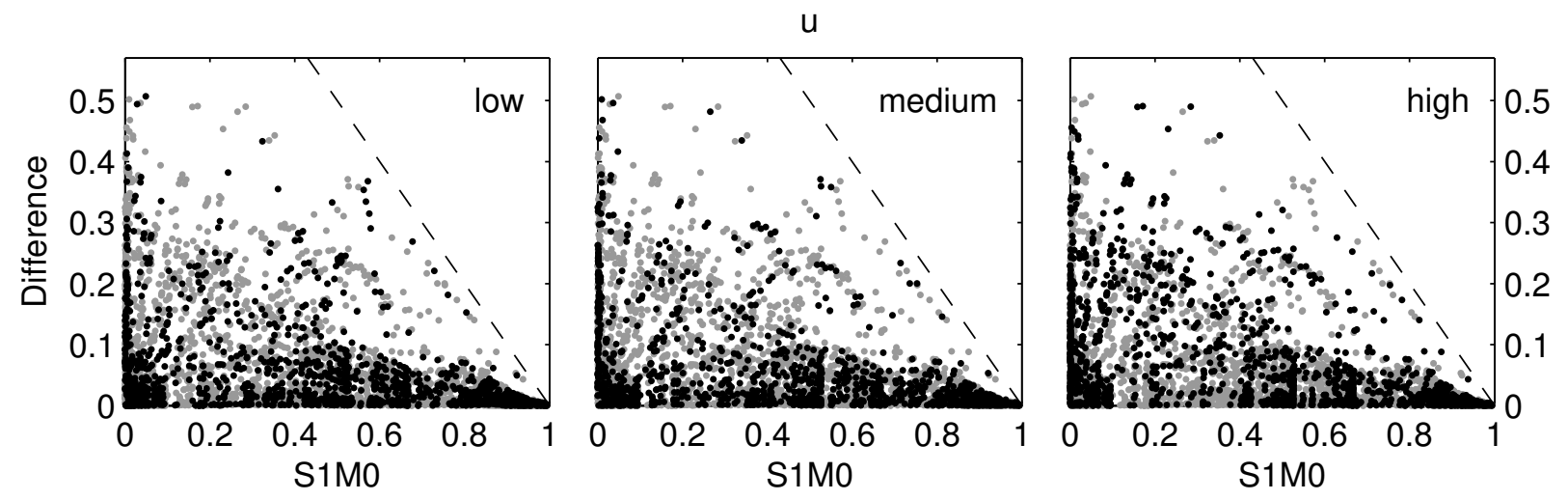

Figure 6: The distribution of differences for parameter $u$. See caption to Figure 4. 

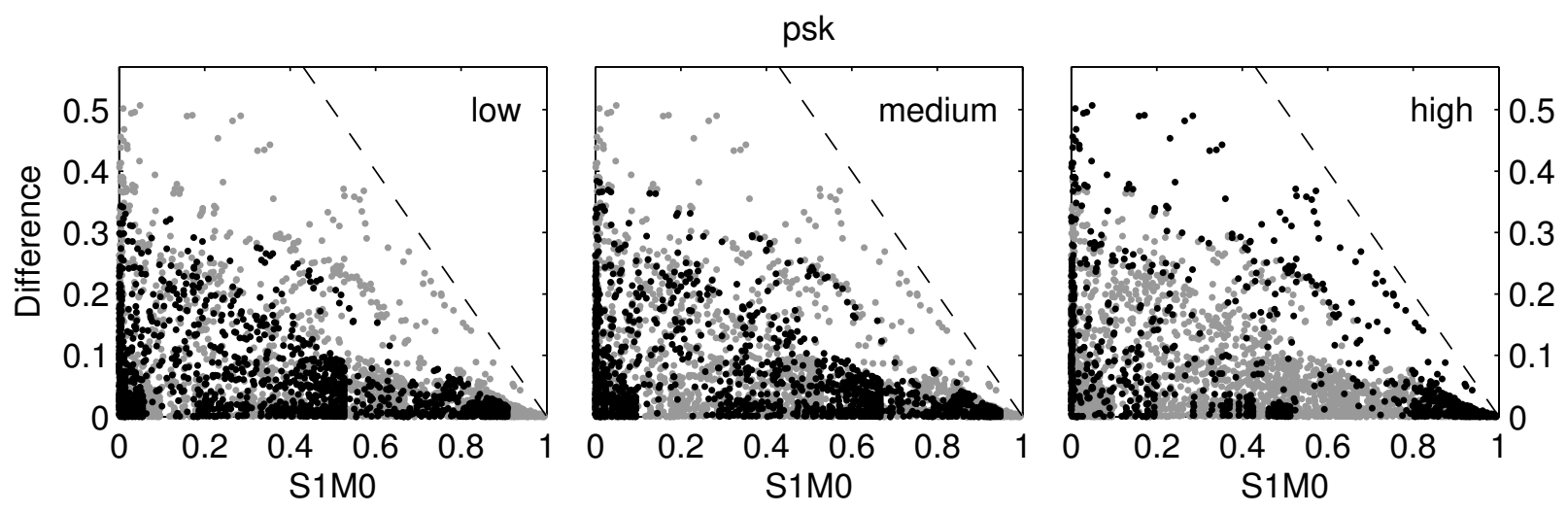

Figure 7: The distribution of differences for parameter $\kappa$. See caption to Figure 4.
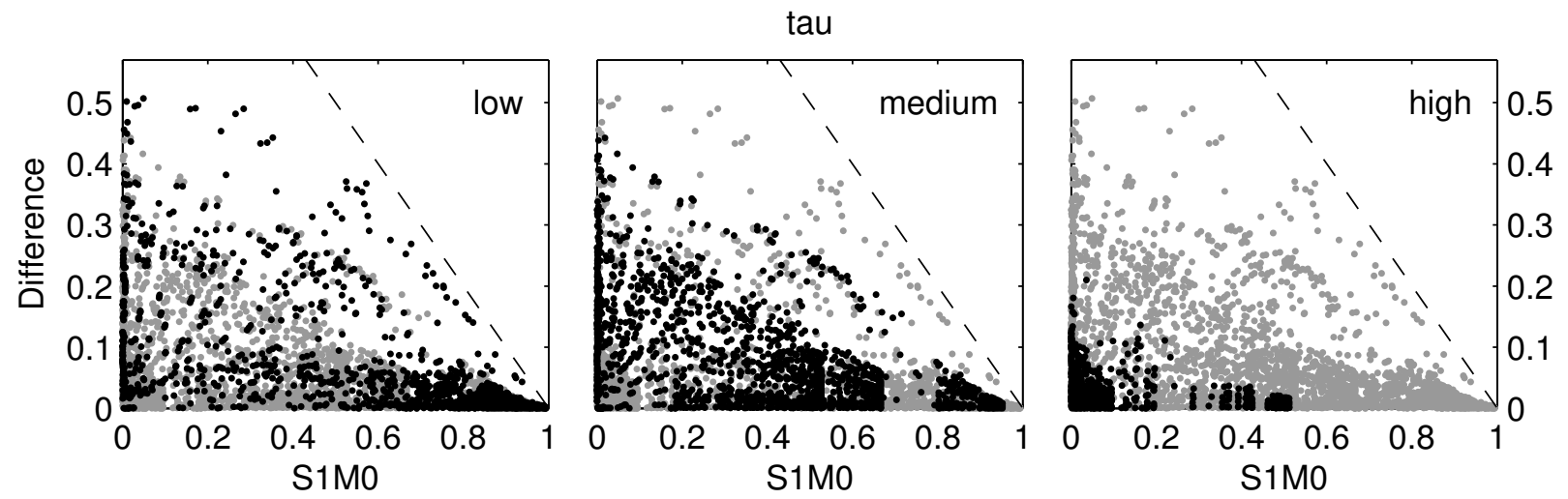

Figure 8: The distribution of differences for parameter $\tau$. See caption to Figure 4. 


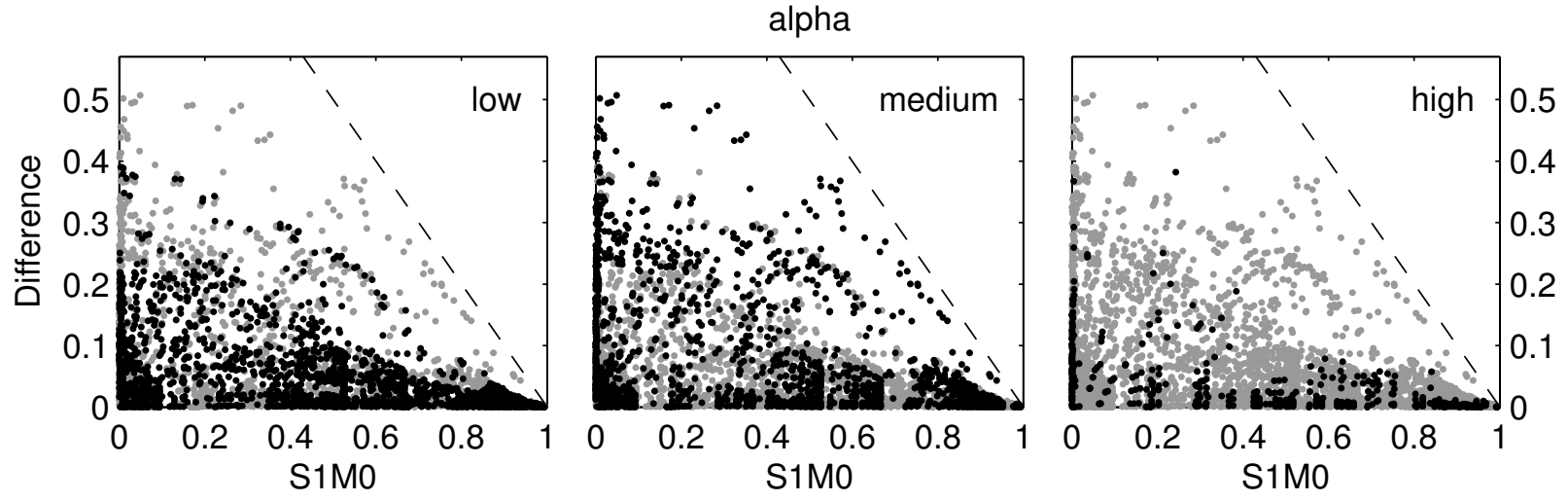

Figure 9: The distribution of differences for parameter $\alpha$. See caption to Figure 4.
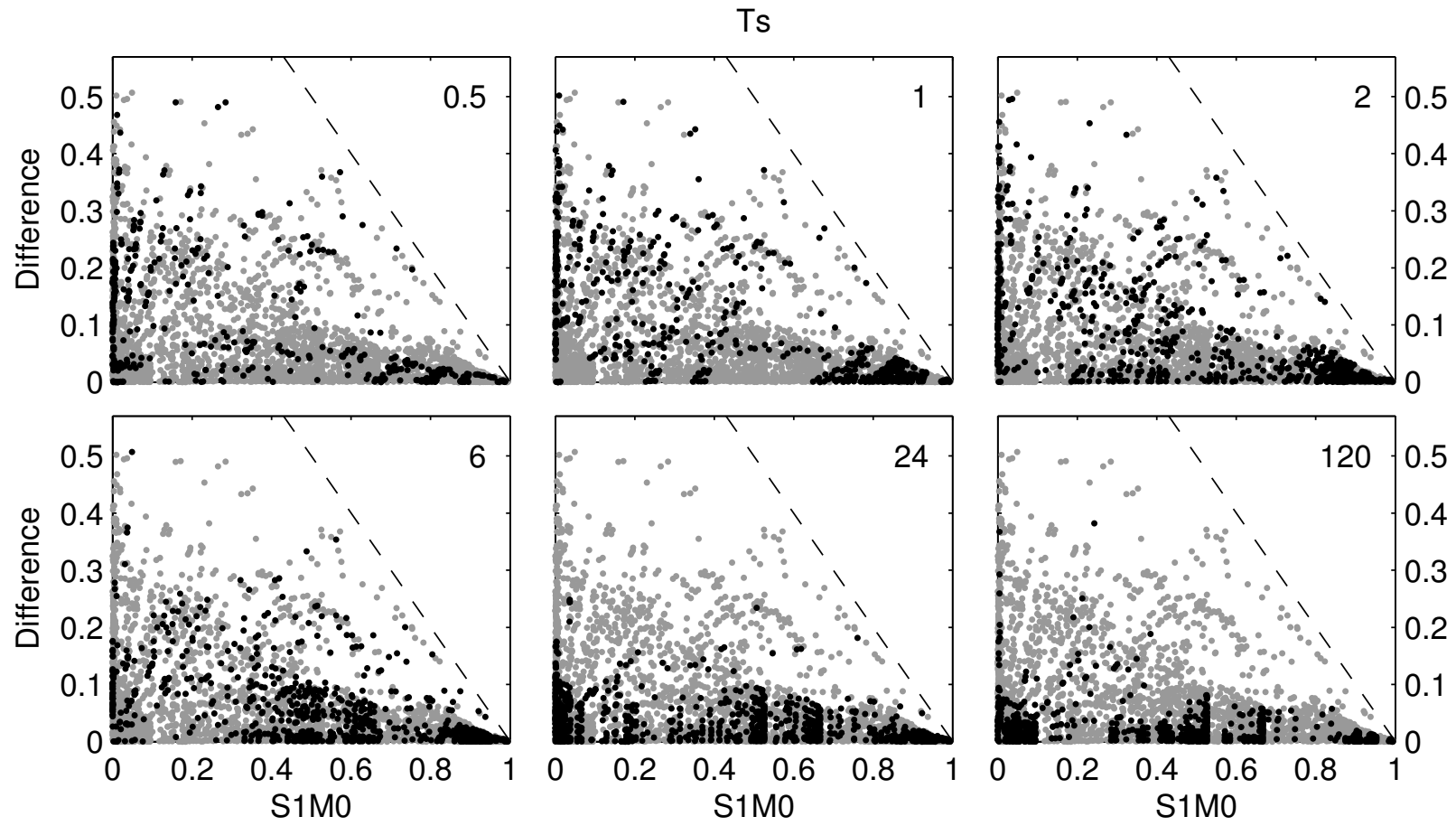

Figure 10: The distribution of differences for parameter $T_{s}$. In each plot, the black dots are for the value of the parameter given in the upper right corner. See caption to Figure 4. 


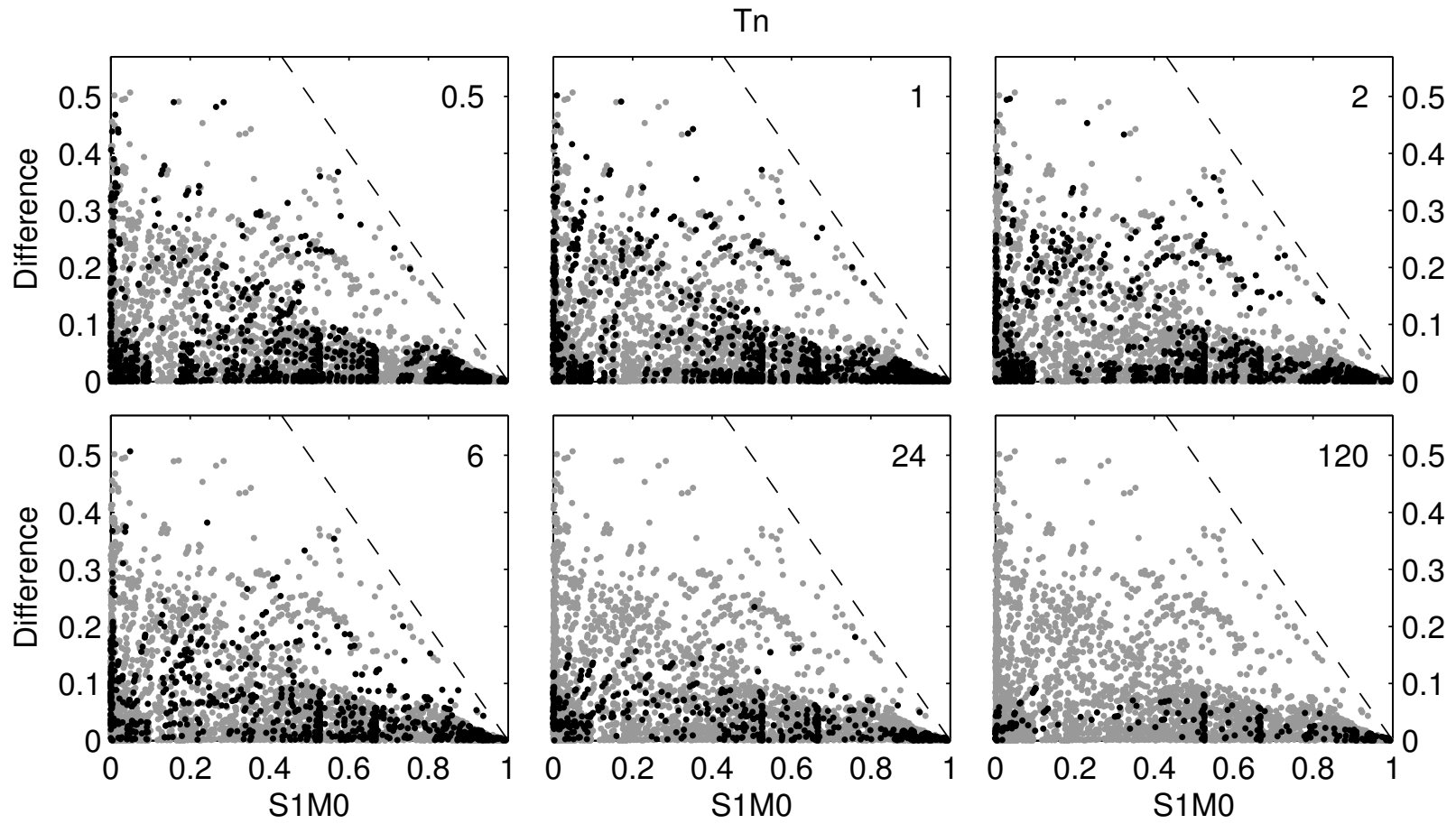

Figure 11: The distribution of differences for parameter $T_{n}$. See caption to Figure 10. 
are not surprising in that if the post-segregational killing mechanism is not very effective or if the missegregation rate is high, the advantage to the plasmid bearer under selection would be severely mitigated. This would result in very low values for the plasmid even under selection, and therefore very little conjugation potential, meaning that models $M_{+}$and $M_{0}$ would not differ substantially in their outcomes. Although smaller values of $T_{s}$ tended to give larger success measure differences between the two models, Figure 10 shows that even at large values of $T_{s}$, there are situations where $S_{1}$ is more than 0.1 greater for model $M_{+}$ over $M_{0}$. It is not surprising that long durations of the non-selection regime ( $T_{n}$ is large) tend to provide model $M_{+}$with little advantage. In this situation the system is driven very close to its equilibrium value during the non-selection regime, that is, $P$ is driven close to zero, so that very little conjugation potential is available during the subsequent selection regime, resulting in little difference between the two models. In contrast, the conditions on $\gamma$ and $\alpha$ are surprising. Intuitively one might expect to see the greatest advantage awarded by the presence of static recipient $F_{+}$cells in situations where the conjugation rate is high and the plasmid cost of carriage is low, so that plasmid-bearing cells have a more significant growth advantage under CM. However, we found that sometimes moderate values of the conjugation rate (Figure 5) and always moderate values of the carriage cost translated to the most significant gains for $M_{+}$over $M_{0}$ (Figure 9). The parameter $u$ is more evenly spread across its relative values with respect to the differences obtained (Figure 6).

Figure 12 shows the relative frequency of low, medium, and high values for each parameter as a function of the success measure difference, $S_{1}\left(M_{+}\right)-S_{1}\left(M_{0}\right)$. To generate this figure, we divided the range of differences, $S_{1}\left(M_{+}\right)-S_{1}\left(M_{0}\right)$, into 6 equal bins and then, for each bin and each parameter, determined the number of times the parameter occurred as a low, medium, or high value divided by the total number of entries in the bin. Again, this figure makes it clear that (i) low values of the conjugation rate, $\gamma$, never occur when the differences in $S_{1}$ are large, (ii) high values of the post-segregational efficiency, $\kappa$, are more common at very large values of $S_{1}\left(M_{+}\right)-S_{1}\left(M_{0}\right)$ but this is not true at lower values of the success difference, (iii) missegregation, $\tau$, is predominantly low or medium when differences are large, (iv) the cost of plasmid carriage, $\alpha$, is medium or low when differences are large, and (v) long regime lengths, $T_{n}$ and $T_{s}$, result in smaller success differences. From the figure it is also evident that when $S_{1}\left(M_{+}\right)-S_{1}\left(M_{0}\right)$ is close to zero, each parameter is more evenly distributed across its possible values. This is due to the fact that very large numbers of the parameter sets led to situations where there was little or no difference in the two models.

Figure 13 shows how the gains in $S_{1}$ are achieved by model $M_{+}$. Predominantly, the increase of $S_{1}\left(M_{+}\right)$over $S_{1}\left(M_{0}\right)$ is achieved by an increase in the value of $P$ by some amount

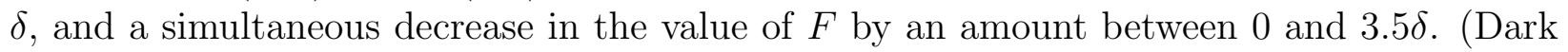
points in wedge-shaped region of Figure 13.) For differences in the success measure between 0.1 and 0.2 the difference was sometimes (5.2\% of the time) achieved by increasing both $F$ and $P$ or (9.7\% of the time) large decreases in $F$. For very small differences, less than 0.1 , increasing both $F$ and $P$ became a more common way (16.9\% of the time) of achieving the 

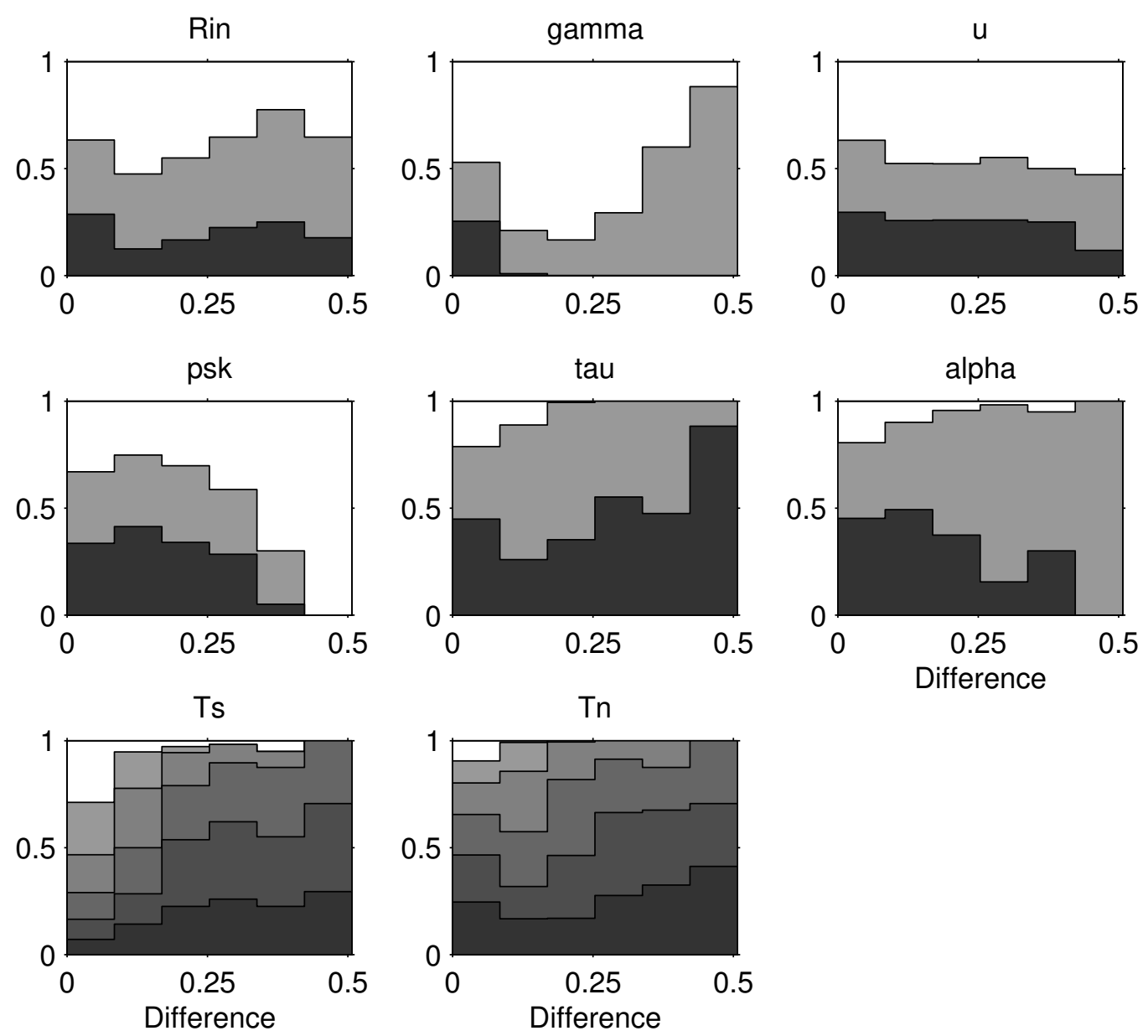

Figure 12: Relative frequency of each parameter value as a function of $S_{1}\left(M_{+}\right)-S_{1}\left(M_{0}\right)$. Each plot is for the named parameter. White indicates the highest value of the parameter and darker colors are for progressively lower values. The vertical range of each color indicates the relative frequency of that parameter value for the given value of $S_{1}\left(M_{+}\right)-S_{1}\left(M_{0}\right)$ (the abscissa). 

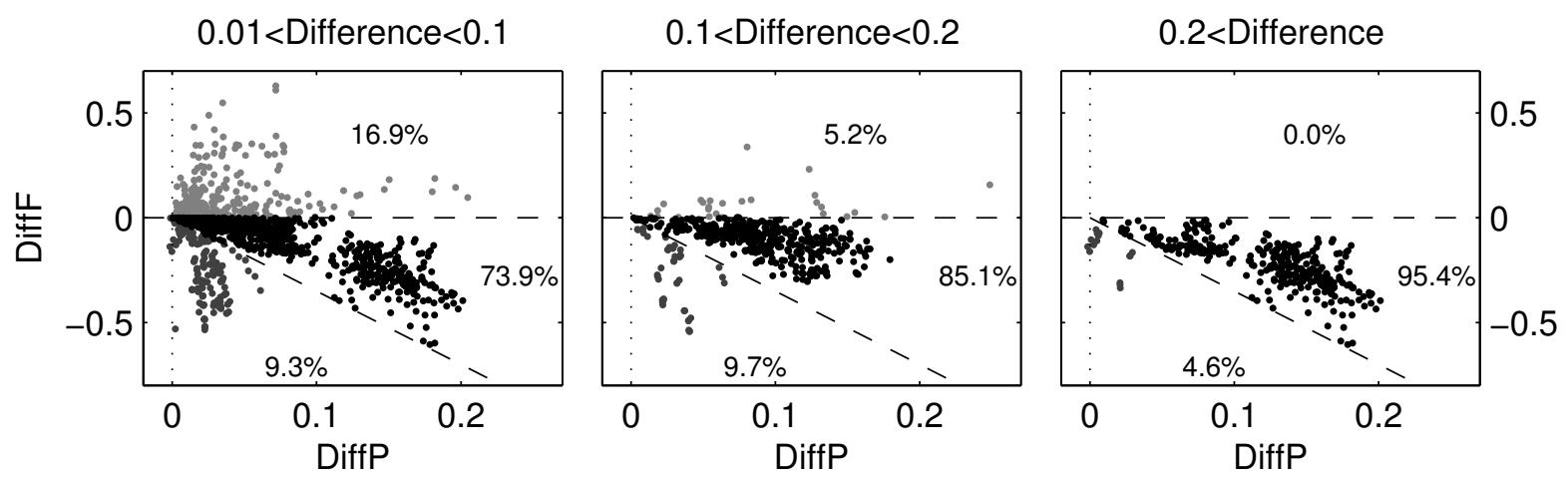

Figure 13: Differences in the values of $P$ and $F$ between models $M_{+}$and $M_{0}$ at the beginning of the stable oscillation cycle. The horizontal axis is the value of $P$ for model $M_{+}$minus its value for model $M_{0}$; the vertical axis is similar but for $F$. The three plots show data for the given ranges of $\Delta S_{1}=S_{1}\left(M_{+}\right)-S_{1}\left(M_{0}\right)$. The data is divided into three regions bordered by the dashed lines with different shadings in each region. The relative frequencies of lying in each region are given.

difference.

\section{Discussion}

In our model we have specifically included a class of cells which have received via conjugation an antibiotic-resistance-bearing plasmid while being exposed to a bacteriostatic agent. These cells are thus static recipients, prevented from expressing these newly acquired genes until the antibiotic is removed (Heinemann, 1998; Wiuff et al., 2005). Our results indicate that this ability to harbour plasmids in "dead" cells can provide a significant increase in plasmid levels in fluctuating selection environments. In some cases, the inclusion of this mechanism allows for persistence of the plasmid when it otherwise would be driven to extinction. Conditions where antibiotic inhibition is not irreversible, such as during exposure to bacteriostatic antibiotics or to low concentrations of bactericidal antibiotics, are the types of environments that may be encountered at certain positions within a biofilm or when pathogens are internalized into human cells, (Ferguson et al., 2002) and at the wane of the antibiotic when a patient stops taking the drug too early.

During antibiotic treatment, a large subpopulation of disease-causing bacteria may become physiologically resistant to the effects of the antibiotic (Wiuff et al., 2005). This subpopulation would also serve as an ideal reservoir for resistance plasmids donated to it from near-by non-disease causing bacteria. Physiological adaptation would also increase the 
chances of surviving long enough to acquire genotypic resistance. In effect, however, this subpopulation is analogous to reinfection during therapy by an already resistant strain rather than being caused by the changing antibiotic concentrations in the patient. For that reason, it is not directly pertinent to our model.

Bacteria can receive conjugative plasmids from donors that have been killed or inhibited for cell division by antibiotics that do not lyse the cell (Heinemann, 1999). If the membrane potential is intact, these same recipients may be converted into donors so long as they can express the genes on the newly acquired plasmid (Debbia, 1992; Heinemann, 1999; Heinemann and Ankenbauer, 1993a,b; Heinemann et al., 1996). Both the ability for "dead" cells to be donors of plasmids and recipients to be able to express resistance genes from newly acquired plasmids while under selection are things which we have not modeled specifically here and which would further increase plasmid persistence.

Our exploration of parameter space comprised a fairly large number of sets, but even so, could not be considered a fine cover. We included values of the parameters near their range extremes, and for those with no upper bound $\left(R_{\mathrm{in}}, T_{s}\right.$, and $\left.T_{n}\right)$ we included what we believe to be large enough values to exhibit the behaviour of the system as the parameter becomes unbounded. The fact that our plots of success differences had a fairly well-defined upper limit and were relatively dense in the interior is a sign that our coverage was representative of all the behaviour possible. We expect that if we had included more intermediate values of the parameters, our success difference plots would have filled out their interiors more fully.

The model studies show that all but the longest culture times in the absence of CM (high value for $T_{n}$ ) allowed for large (greater than 0.1 ) increases in the plasmid levels when the plasmid could transfer to the cell division-arrested population (i.e., $F_{+}$cells were included). The frequency of significant increases was reduced when $T_{n}$ or $T_{s}$ were 24 or higher. The nondimensional time units are in terms of units of $e$-fold growth under maximal growth conditions. Assuming a generation time of about 1 hour, an $e$-fold increase would occur in about 1.5 hours, meaning the selection/non-selection regimes which yield significant increases in plasmid levels are between 0.75 and 35 hours $\left(T_{n}\right.$ and $T_{s}$ from 0.5 to 24$)$. These times correspond to typical antibiotic dose schedules. Figure 14 shows the distribution of differences in success measure for values of culture times in the presence of $\mathrm{CM}\left(T_{s}\right)$ when $T_{n}=0.5$, corresponding to about three quarters of an hour where the antibiotic is absent between periods of CM presence. The potential for significant gains for the plasmid because of the presence of $F_{+}$cells is more substantial when $T_{s}$ is also small, however, even at $T_{s}=6$, corresponding to about 9 hours of CM presence, $F_{+}$cells can give advantages around 0.1, that is, relative frequencies of plasmid-bearing cells $10 \%$ higher when conjugation to static cells is included. These results indicate that the spread of genes awarding resistance to bacteriostatic agents is enhanced by the presence of cells arrested by the agent but still capable of being a conjugation recipient.

Although our model had instantaneous transitions between the presence and absence of $\mathrm{CM}$, the qualitative results we have obtained are not altered by using a model incorporating 

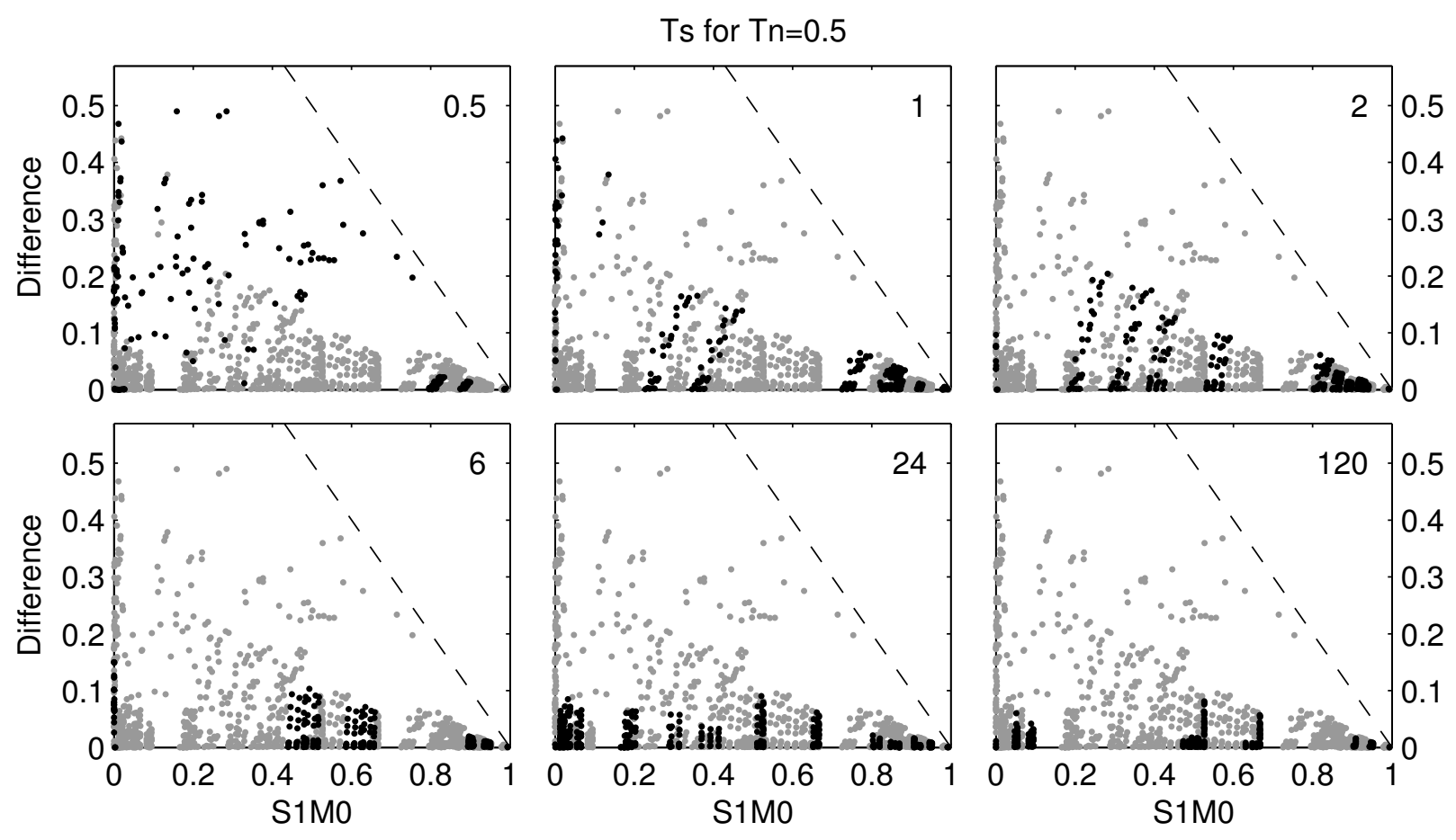

Figure 14: The distribution of differences for parameter $T_{s}$ when $T_{n}=0.5$. In each plot, the black dots are for the value of the parameter given in the upper right corner and the lighter dots in the background are the differences for the other values of $T_{s}$. The dashed line is the maximum possible difference, since $S_{1}\left(M_{+}\right)$cannot exceed one. 
a smooth transition period between inhibitory and non-inhibitory CM concentrations when $\mathrm{CM}$ is partially active. One reasonable way of modeling the slow removal/dilution of CM from the system would be to insert a transition regime of length $T_{t}$ after the selection regime, during which the governing system of equations is

$$
\text { CM removal }\left\{\begin{aligned}
\frac{d P}{d t} & =\left(\psi(R)(1-\alpha)(1-\tau)-u+\gamma F h_{1}(t)\right) P+h_{2}(t) F_{+} \\
\frac{d F}{d t} & =\left(\psi(R) h_{1}(t)-u-\gamma P\right) F+\psi(R)(1-\alpha) \tau(1-\kappa) P \\
\frac{d F_{+}}{d t} & =-u F_{+}+\gamma F\left(1-h_{1}(t)\right) P-h_{2}(t) F_{+} \\
\frac{d R}{d t} & =\left(R_{\text {in }}-R\right) u-\frac{1}{\sigma} \psi(R)\left(P+F h_{1}(t)\right),
\end{aligned}\right.
$$

where $h_{1}(t)$ and $h_{2}(t)$ are monotonic increasing functions mapping the transition time interval onto $(0,1)$ and $(0, \infty)$ respectively. The function $h_{1}(t)$ represents the fraction of plasmid-free cells that are growing at low CM concentrations, and the function $h_{2}(t)$ is the conversion rate of $F_{+}$cells to $P$ cells, which, since it is unbounded as $t$ approaches the end of the transition regime, ensures all $F_{+}$cells convert to $P$ cells within the time span $T_{t}$. A similar set of equations could be used to model a slow increase in CM concentration between the nonselection and selection regimes, where now $h_{1}$ and $h_{2}$ are monotonic decreasing functions mapping to their ranges. In the limit as $T_{t} \rightarrow 0$, this transition model approaches the instantaneous model we have employed. Similarly, we could relax the effect of CM during the selection regime allowing for some growth of the $F$ cell population and a growth reduction of the $P$ cell population. For all of these changes, we expect that our qualitative results will still hold: there will be some parameter sets for which inclusion of $F_{+}$cells in the model allow persistence of the plasmid when their exclusion does not, and other sets for which the inclusion of $F_{+}$cells significantly increases the relative frequency of plasmid-bearers in the system.

The enhancing effect of static recipient cells was unaffected by the value of the wash out rate, $u$, indicating that the flushing rate is unimportant. The input resource concentration, $R_{\text {in }}$, and the PSK efficiency, $\kappa$, allowed substantial gains in plasmid persistence at any of their values but the largest gains occurred when $R_{\text {in }}$ was moderate to high and $\kappa$ was high. Longer regime times, $T_{n}$ and $T_{s}$, muted the effect of including static recipient cells since, if $T_{n}$ is large the plasmid-bearing cells, and if $T_{s}$ is large the plasmid-free cells, are driven to or near extinction due to compromised growth. High missegregation rates, $\tau$, and low conjugation rates, $\gamma$, gave very little ability for the static cells to enhance plasmid persistence. However, high and (surprisingly) moderate values of $\gamma$ (as low as half the rate needed for the plasmid to persist on its own as a parasite), allowed for large increases in the relative frequency of the plasmid. Similarly, although intuitively one might expect that static recipient cells would 
have the most effect on plasmid numbers in cases where the cost of plasmid carriage, $\alpha$, is small, moderate values (so that plasmid-bearers grew at only half the rate of plasmid-free cells) yielded the largest gains for the plasmid.

Throughout this paper we have used values of the cells after a stable oscillation had been reached in a continuously cycling environment of selection and nonselection. Admittedly such strictly regimented environments likely do not occur. However, in many of our simulations, the effect of including $F_{+}$cells was even more pronounced during the transient phase before solutions had settled to the stable oscillation. This means that the static recipient mechanism is likely more important in increasing plasmid frequency in situations where only a few cycles of antibiotic presence and absence is encountered. In order to accurately quantify the effect of static recipients in this type of transient regime, the measure would have to take into account the initial conditions of the system. Further, some type of cycling environment would still be required because $F_{+}$cells only become important once the bacteriostatic agent has been removed. We believe that our results here shed some light on the importance of static recipient cells for the persistence of plasmids encoding resistance to bacteriostatic agents.

\section{Appendix}

Here we establish the global stability of equilibrium (9) for system (2a) and provide evidence for the global stability of equilibrium (11) for system (2b). We also show that throughout the stable oscillation cycle we have $P+F+F_{+} \leq 1$. We assume that $\alpha<1$ (carriage cost does not eliminate all growth), $\tau<1$ (missegregation does not always occur), and $\kappa<1$ (post-segregational killing is not completely effective), although we also comment on the case $\kappa=1$.

\section{A.1 No CM}

First consider system (2a). Let $x=(P, F, R)$ and $D$ be the nonnegative orthant for $x$. Define $z=P+F+\sigma R$ and $\Omega=\left\{x \in D \mid z \leq \sigma R_{\text {in }}\right\}$, where $\sigma$ is given by (6). See Figure 15 . We have

$$
\left.\frac{d P}{d t}\right|_{P=0}=0,\left.\quad \frac{d F}{d t}\right|_{F=0} \geq 0,\left.\quad \frac{d R}{d t}\right|_{R=0} \geq 0 .
$$

This establishes the positive invariance of $D$ and the $P=0$ plane. Further, a simple computation shows that

$$
\frac{d z}{d t}=\left(\sigma R_{\text {in }}-z\right) u-\psi(R)[1-(1-\alpha)(1-\tau \kappa)] P
$$

so that $\left.\frac{d z}{d t}\right|_{z=\sigma R_{\text {in }}} \leq 0$. This establishes the positive invariance of the region $\Omega$. In addition, from (14) it is clear that $\left.\frac{d z}{d t}\right|_{z>\sigma R_{\mathrm{in}}}<0$. Thus all points in $D$ either enter $\Omega$ or they may 


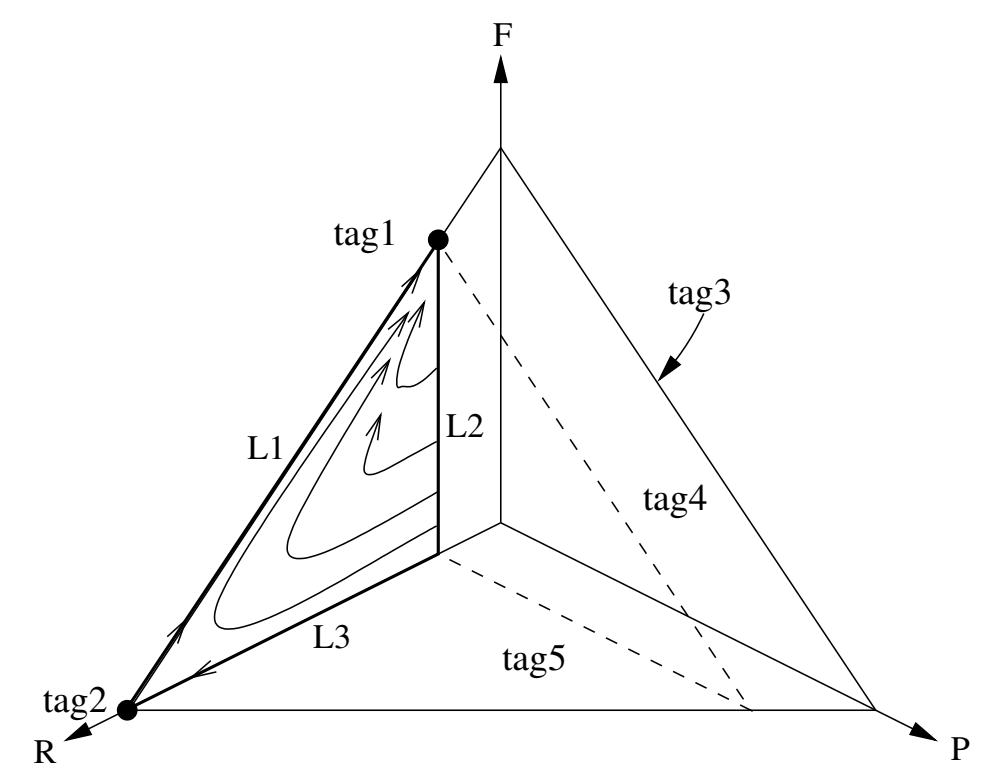

Figure 15: Geometry of system (2a). $\Omega$ is the tetrahedron in the nonnegative orthant comprised of regions $\Omega_{1}$ and $\Omega_{2}$ which are separated by the dashed line triangle. All trajectories approach the triangle in the $P=0$ plane bounded by the line segments $L_{1}, L_{2}$ and $L_{3}$, drawn in bold. The qualitative dynamics on this triangle are sketched; all solutions on the triangle approach the equilibrium $e_{2}$ except those on $L_{3}$ which approach the saddle point $e_{1}$. 
remain outside $\Omega$ for all time but approach the $z=\sigma R_{\text {in }}$ boundary of $\Omega$ as $t \rightarrow \infty$. In the latter case, $\frac{d z}{d t}$ must also approach zero, so that, from (14) we see that either $P \rightarrow 0$ or $R \rightarrow 0$. (Recall that $\psi(\cdot)$ is a positive definite function with respect to zero.) However, if $R \rightarrow 0$, then $\frac{d R}{d t} \rightarrow R_{\text {in }} u$ which is a finite positive number generating a contradiction. We conclude that all points either enter the positively invariant region $\Omega$ or they remain outside $\Omega$ but approach the line segment $L_{1}=\left\{x \in \Omega \mid P=0, F+\sigma R=R_{\text {in }}\right\}$ as $t \rightarrow \infty$.

Define the sets

$$
\Omega_{1}=\{x \in \Omega \mid u>\psi(R)\} \quad \text { and } \quad \Omega_{2}=\{x \in \Omega \mid u \leq \psi(R)\} .
$$

For $x \in \Omega$ we have $P+F \leq \sigma\left(R_{\text {in }}-R\right)$ so that

$$
\frac{d R}{d t}=\left(R_{\text {in }}-R\right) u-\frac{\psi(R)}{\sigma}(P+F) \geq\left(R_{\text {in }}-R\right)(u-\psi(R)) .
$$

Therefore all points in $\Omega_{1}$ have $R$ increasing so that they eventually either enter $\Omega_{2}$ (which is also positively invariant) or they remain in $\Omega_{1}$ but approach the boundary $R=\psi^{-1}(u)$ of $\Omega_{2}$ as $t \rightarrow \infty$. In the latter case,

$$
\frac{d P}{d t} \rightarrow[\gamma F-u(1-(1-\alpha)(1-\tau))]
$$

but $F \leq 1$ on this boundary (see Figure (15)), so by (10) $P$ is eventually decreasing. We conclude that all points in $\Omega$ either enter the positively invariant region $\Omega_{2}$ or they remain in $\Omega_{1}$ but approach the line segment $L_{2}=\left\{x \in \Omega \mid P=0,0 \leq F \leq 1, R=\psi^{-1}(u)\right\}$ as $t \rightarrow \infty$.

For $x \in \Omega_{2}, F \neq 0$, we have

$$
\begin{aligned}
\frac{d\left(\frac{P}{F}\right)}{d t} & =\frac{P}{F}\left(-\psi(R)[1-(1-\alpha)(1-\tau)]+\gamma(F+P)-\psi(R)(1-\alpha) \tau(1-\kappa) \frac{P}{F}\right) \\
& <\frac{P}{F}\left([1-(1-\alpha)(1-\tau)][-\psi(R)+u(F+P)]-\psi(R)(1-\alpha) \tau(1-\kappa) \frac{P}{F}\right) \\
& \leq \frac{P}{F}\left([1-(1-\alpha)(1-\tau)]\left[u \sigma\left(R_{\text {in }}-R\right)-\psi(R)\right]-\psi(R)(1-\alpha) \tau(1-\kappa) \frac{P}{F}\right)
\end{aligned}
$$

where we have used (10) and the fact that $P+F \leq \sigma\left(R_{\text {in }}-R\right)$ in $\Omega$. Define

$$
f(R)=u \sigma\left(R_{\mathrm{in}}-R\right)-\psi(R) .
$$

Since $\psi(R)$ is a monotonic increasing function, it follows that $f(R)$ is monotonic decreasing. Further, since $\sigma=\left(R_{\text {in }}-\psi^{-1}(u)\right)^{-1}$, it follows that $f(R)$ is negative for $R>\psi^{-1}(u)$. Thus, in $\Omega_{2}$, with $F \neq 0$, we have $f(R) \leq 0$ and we conclude that $d\left(\frac{P}{F}\right) / d t<0$. If $F=0$ in $\Omega_{2}$ then $F$ will increase unless $P$ is also zero. Therefore all points in $\Omega_{2}$ will approach the $P=0$ 
boundary of $\Omega_{2}$ (a triangle in the $\left(F, R\right.$ )-plane bounded by $L_{1}, L_{2}$ and the line segment $\left.L_{3}=\left\{x \in \Omega \mid P=0, F=0, \psi^{-1}(u) \leq R \leq R_{\text {in }}\right\}\right)$.

This triangle lies in the invariant $P=0$ plane. The only equilibria of (2a) on this triangle are

$$
e_{1}=\left(0,0, R_{\text {in }}\right) \text { and } e_{2}=\left(0,1, \psi^{-1}(u)\right)
$$

which are located at the two end points of the line segment $L_{1}$. In the $P=0$ plane, the normal to $L_{1}$ is $(1, \sigma)$. A quick calculation shows that this normal is orthogonal to the vector field on $L_{1}$ and so we conclude that $L_{1}$ is invariant, that is, it is comprised of the two equilibria $e_{1}$ and $e_{2}$, and a trajectory joining them. Since a closed orbit on the plane must enclose an equilibrium, we conclude that no closed orbits can exist on this boundary of $\Omega_{2}$. The eigenvalues of the Jacobian (in $(P, F, R)$-space) at the equilibrium point $e_{1}$ are

$$
-u, \quad,-u+(1-\alpha)(1-\tau) \psi\left(R_{\mathrm{in}}\right), \text { and }-u+\psi\left(R_{\mathrm{in}}\right),
$$

the latter two of which are positive, by (12). The stable manifold of the saddle point $e_{1}$ is the $R$-axis. The eigenvalues of the Jacobian at $e_{2}$ are

$$
-u,-u[1-(1-\alpha)(1-\tau)]+\gamma, \text { and }-u(1-u)\left(R_{\text {in }}-\psi^{-1}(u)\right)\left(\frac{1}{u}-1\right),
$$

which by (10) and (12) are all negative. Thus $e_{2}$ is locally asymptotically stable. Since we have already excluded closed orbits on this boundary of $\Omega_{2}$, we conclude that all orbits on the triangle less those on $L_{3}$ approach $e_{2}$ as $t \rightarrow \infty$.

In summary then, all orbits starting in $D$ are approaching some part of the closed triangle on the $P=0$ plane bounded by the line segments $L_{1}, L_{2}$ and $L_{3}$, and all orbits on the triangle except those on $L_{3}$ are approaching the equilibrium $e_{2}$ located at the intersection of $L_{1}$ and $L_{2}$. Points on $L_{3}$ approach $e_{1}$ located at the intersection of $L_{1}$ and $L_{3}$. Since the stable manifold of $e_{1}$ in $(P, F, R)$-space is the $R$-axis, we conclude that the only points which approach $e_{1}$ are those with $P=0$ and $F=0$ (the uninteresting trivial case where there is no bacteria ever). By continuity of solutions of the differential equations then, we conclude that $e_{2}$ is globally asymptotically stable in $D$ less the $R$-axis.

If $\kappa=1$ (post-segregational killing is completely effective) the $(P, R)$-plane is invariant (corresponding to the situation where all bacteria are plasmid bearers and post-segregational killing prevents the emergence of any viable free cells when missegregation events occur). In this case, the system has another equilibrium located at

$$
e_{3}=\left((1-\alpha)(1-\tau) \frac{R_{\mathrm{in}}-\psi^{-1}\left(\frac{u}{(1-\alpha)(1-\tau)}\right)}{R_{\mathrm{in}}-\psi^{-1}(u)}, 0, \psi^{-1}\left(\frac{u}{(1-\alpha)(1-\tau)}\right)\right)
$$

This equilibrium is located on the interior of the triangle which is the $F=0$, boundary of $\Omega_{2}$. A linear stability analysis shows that $e_{3}$ is locally stable in the $(P, R)$-plane and unstable 
in a direction transverse to the plane when (10) and (12) hold. Thus if PSK was truly one hundred percent effective we would expect system (2a) to approach $e_{3}$ rather than $e_{2}$ as $t \rightarrow \infty$. However, any small introduction of $F$ cells will place the system into the situation described previously and hence it will approach $e_{2}$.

\section{A.2 CM}

The analysis for equilibrium (11) of system (2b) is similar to that given above; we highlight the differences below. Briefly, they are as follows. In this case we can show that all trajectories starting in $D$ (less the $R$-axis) definitely enter $\Omega_{2}$. However, although we can establish that there are just two equilibria in $\Omega_{2}$ only one of which is locally stable, proving global stability is more difficult. Nonetheless, numerical evidence indicates that there are no other attractors.

We define $x=\left(P, F, F_{+}, R\right)$, and $z=P+F+F_{+}+\sigma R$. As before, $D$ is positively invariant and $\frac{d z}{d t}$ is still given by (14). Hence by the same argument as for the no CM case, for a trajectory to remain outside $\Omega$ it is necessary that $P \rightarrow 0$ as $t \rightarrow \infty$. However, from (2b) we see that as $P \rightarrow 0, R$ will approach $R_{\text {in }}$ and thus using (12) we have

$$
\frac{d P}{d t} \rightarrow \psi\left(R_{\text {in }}\right)(1-\alpha)(1-\tau) P-u P>0,
$$

indicating that $P$ cannot decrease to zero. Therefore all trajectories enter $\Omega$.

Once inside $\Omega$ we have

$$
\frac{d R}{d t}=\left(R_{\mathrm{in}}-R\right) u-\frac{\psi(R)}{\sigma} P \geq\left(R_{\mathrm{in}}-R\right)(u-\psi(R))+\frac{\psi(R)}{\sigma}\left(F+F_{+}\right),
$$

so that for $x \in \Omega_{1}, \frac{d R}{d t}>0$. We conclude that either trajectories enter $\Omega_{2}$ or they remain in $\Omega_{1}$ but approach $R=\psi^{-1}(u)$ and have $\frac{d R}{d t} \rightarrow 0$ as $t \rightarrow \infty$. This last requirement would mean that both $F$ and $F_{+}$approach zero but then $\frac{d F}{d t} \rightarrow \psi(R)(1-\alpha) \tau(1-\kappa) P$ which would also have to be zero meaning that $P \rightarrow 0$. The same argument as above shows that $P$ cannot approach zero and so we conclude that points must enter $\Omega_{2}$.

There are just two equilibria in $\Omega_{2}$ for system (2b):

$$
\begin{aligned}
e_{4}= & \left(0,0,0, R_{\mathrm{in}}\right) \\
e_{5}= & \left((1-\alpha)(1-\tau) \frac{R_{\mathrm{in}}-\psi^{-1}\left(\frac{u}{(1-\alpha)(1-\tau)}\right)}{R_{\mathrm{in}}-\psi^{-1}(u)}, \frac{(1-\kappa) \tau P}{(1-\tau)\left(1+\frac{\gamma}{u} P\right)},\right. \\
& \left.\quad \frac{\gamma F P}{u}, \psi^{-1}\left(\frac{u}{(1-\alpha)(1-\tau)}\right)\right)
\end{aligned}
$$


One eigenvalue of the Jacobian at $e_{4}$ is $-u+(1-\alpha)(1-\tau) \psi\left(R_{\text {in }}\right)$ which is positive due to (12). The eigenvalues of the Jacobian at $e_{5}$ are $-u$ (twice), $-u-\gamma P$, and

$$
-\frac{\left(R_{\text {in }}-\psi^{-1}\left(\frac{u}{(1-\alpha)(1-\tau)}\right)\right) u^{2}}{(1-\alpha)(1-\tau)\left(\psi^{-1}\left(\frac{u}{(1-\alpha)(1-\tau)}\right)\right)^{2}},
$$

the first three of which are clearly negative while the last is negative by (12). Thus $e_{4}$ is unstable and $e_{5}$ is locally asymptotically stable. Proving global stability of $e_{5}$ is more difficult. Showing that there are no other attractors in $\Omega_{2}$ is not straight forward, but numerical evidence indicates that $e_{5}$ is globally stable.

\section{A.3 Normalized Cell Count}

Consider the full alternating system which switches between the non-selection regime, (2a), and the selection regime, $(2 \mathrm{~b})$. The transformation between the two regimes $\left(F_{+}\right.$cells become $P$ cells when CM is removed, $F_{+}$is initialized as zero when $C M$ is added) leave $z$ and $R$ invariant. Thus both $\Omega$ and $\Omega_{2}$ are positively invariant under the full alternating system. If the full alternating system has a stable oscillating solution then this solution must necessarily have the same values for $z$ and $R$ at the start of each full cycle. Since $\frac{d z}{d t}$ is strictly decreasing outside $\Omega$ under both regimes, we conclude that this stable oscillating solution cannot be entirely outside $\Omega$. Thus it must enter $\Omega$, and once there, remain in this positively invariant set. Similarly, since $\frac{d R}{d t}$ is strictly increasing in $\Omega_{1}$ under both regimes, we conclude that the stable oscillating solution cannot be entirely inside $\Omega_{1}$. Thus it must enter $\Omega_{2}$, and therefore must lie entirely in $\Omega_{2}$. For points in $\Omega_{2}$ we have

$$
P+F+F_{+} \leq \sigma\left(R_{\text {in }}-R\right) \leq \sigma\left(R_{\text {in }}-\psi^{-1}(u)\right)=1,
$$

since $\sigma$ given by $(6)$ is equivalent to $\sigma=\left(R_{\text {in }}-\psi^{-1}(u)\right)^{-1}$. Therefore $P+F+F_{+} \leq 1$ during the entire stable oscillation.

\section{References}

Amábile-Cuevas, C. F. (Ed.), 1996. Antibiotic Resistance: From Molecular Basics to Therapeutic Options. Chapman \& Hall/R.G. Landes, New York.

Bergstrom, C. T., Lipsitch, M., Levin, B. R., 2000. Natural selection, infectious transfer and the existence conditions for bacterial plasmids. Genetics 155, 1505-1519.

Cooper, T. F., Heinemann, J. A., 2000a. Postsegregational killing does not increase plasmid stability but acts to mediate the exclusion of competing plasmids. Proc. Natl. Acad. Sci. USA 97, 12543-12648. 
Cooper, T. F., Heinemann, J. A., 2000b. Transfer of conjugative plasmids and bacteriophage occurs in the presence of antibiotics that prevent de novo gene expression. Plasmid 43, $171-175$.

Cooper, T. F., Heinemann, J. A., 2005. Selection for plasmid postsegregational killing depends on multiple infection: Evidence for the selection of more virulent parasites through parasite-level competition. Proc. Roy. Soc. Lon. B 272, 403-410.

Debbia, E. A., 1992. Filamentation promotes F'lac loss in Escherichia coli K12. J. Gen. Microbiol. 138, 2083-2091.

Dionisio, F., Matic, I., Radman, M., Rodrigues, O. R., Taddei, F., 2002. Plasmids spread very fast in heterogeneous bacterial communities. Genetics 162, 1525-1532.

Eberhard, W. G., 1989. Why do bacterial plasmids carry some genes and not others? Plasmid $21,167-174$.

Ferguson, G. C., Heinemann, J. A., Kennedy, M. A., 2002. Gene transfer between Salmonella enterica serovar typhimurium inside epithelial cells. J. Bacteriol. 184, 2235-2242.

Frost, L. S., 2000. Conjugation, bacterial. In: Lederberg, J. (Ed.), Encyclopedia of Microbiology. Academic Press, San Diego, CA, pp. 847-862.

Gerdes, K., Rasmussen, P. B., Molin, S., 1986. Unique type of plasmid maintenance function: Postsegregational killing of plasmid-free cells. Proc. Natl. Acad. Sci. USA 83, 3116-3120.

Ghigo, J.-M., 2001. Natural conjugative plasmids induce bacterial biofilm development. Nature $412,442-445$.

Gillespie, S. H., McHugh, T. S., 1997. The biological cost of antimicrobial resistance. Trends Microbiol. 5, 337-339.

Gordon, D. M., 1992. Rate of plasmid transfer among Eschericia coli strains isolated from natural populations. J. Gen. Microbiol. 138, 17-21.

Heinemann, J. A., 1991. Genetics of gene transfer between species. Trends Genet. 7, 181-185.

Heinemann, J. A., 1998. Looking sideways at the evolution of replicons. In: Kado, C. I., Syvanen, M. (Eds.), Horizontal Gene Transfer. International Thomson Publishing, London, pp. 11-24.

Heinemann, J. A., 1999. How anitbiotics cause antibiotic resistance. Drug Discov. Today 4, $72-79$. 
Heinemann, J. A., 2000. Complex effects of dna gyrase inhibitors on bacterial conjugation. J. Biochem. Mol. Biol. Biophys. 4, 165-177.

Heinemann, J. A., Ankenbauer, R. G., 1993a. Retrotransfer in Escherichia coli conjugation: Bidirectional exchange or de novo mating? J. Bacteriol. 175, 583-588.

Heinemann, J. A., Ankenbauer, R. G., 1993b. Retrotransfer of incP plasmid R751 from Escherichia coli maxicells: Evidence for the genetic sufficiency of self-transferable plasmids for bacterial conjugation. Mol. Microbiol. 10, 57-62.

Heinemann, J. A., Ankenbauer, R. G., Amabile-Cuevas, C. F., 2000. Do antibiotics maintain antibiotic resistance? Drug Discov. Today 5, 195-204.

Heinemann, J. A., Scott, H. E., Williams, M., 1996. Doing the conjugative two-step: Evidence of recipient autonomy in retrotransfer. Genetics 143, 1425-1435.

Heinemann, J. A., Silby, M. W., 2003. Horizontal gene transfer and the selection of antibiotic resistance. In: Amábile-Cuevas, C. F. (Ed.), Multiple Drug Resistant Bacteria. Horizon Scientific Press, Wymondham, pp. 161-178.

Imran, M., Jones, D., Smith, H., 2005. Biofilms and the plasmid maintenance question. Math. Biosci. 193, 183-204.

Kobayashi, I., 1998. Selfishness and death: Raison d'etre of restriction, recombination and mitochondria. Trends Genet. 14, 368-374.

Lenski, R. E., Simpson, S. C., Nguyen, T. T., 1994. Genetic analysis of a plasmid-encoded, host genotype-specific enhancement of bacterial fitness. J. Bacteriol. 176, 3140-3147.

Levin, B. R., Stewart, F. M., Rice, V. A., 1979. The kinetics of conjugative plasmid transmission: Fit of a simple mass action model. Plasmid 2, 247-260.

Modi, R. I., Casilla, L. H., Puskas-Pozsa, S., Helling, R. B., Adams, J., 1992. Genetic changes accompanying increased fitness in evolving populations of Escherichia coli. Genetics 130, 241-249.

Peters, J. E., Benson, S. A., 1995. Redundant transfer of F' plasmids occurs between Escherichia coli cells during nonlethal selections. J. Bacteriol. 177, 847-850.

Schrag, S. J., Perrot, V., 1996. Reducing antibiotic resistance. Nature 381, 120-121.

Schrag, S. J., Perrot, V., Levin, B. R., 1997. Adaptation to the fitness of antibiotic resistance in Escherichia coli. Proc. Roy. Soc. Lond. B 264, 1287-1291. 
Simonsen, L., 1991. The existence conditions for bacterial plasmids: Theory and reality. Microb. Ecol. 22, 187-205.

Smith, J., 2001. The social evolution of bacterial pathogenesis. Proc. R. Soc. Lond. B 268, 61-69.

Souza, V., Eguiarte, L. E., 1997. Bacteria gone native vs. bacteria gone awry?: Plasmid transfer and bacterial evolution. Proc. Natl. Acad. Sci. USA 94, 5501-5503.

Stewart, F. M., Levin, B. R., 1977. The population biology of bacterial plasmids: a priori conditions for the existence of conjugationally transmitted factors. Genetics 87, 209-228.

Turner, P. E., 2004. Phenotypic plasticity in bacterial plasmids. Genetics 167, 9-20.

Turner, S. L., Bailey, M. J., Lilley, A. K., Thomas, C. M., 2002. Ecological and molecular maintenance strategies of mobile genetic elements. FEMS Microbiol. Ecol. 42, 177-185.

Wiuff, C., Zappala, R. M., Regoes, R. R., Garner, K. N., Baquero, F., Levin, B. R., 2005. Phenotypic tolerance: Antibiotic enrichment of noninherited resistance in bacterial populations. Antimicrob. Agents Chemother. 49, 1483-1494. 